\title{
A Continuation Method for Nash Equilibria in Structured Games
}

\author{
Ben Blum \\ BBLUM@CS.BERKELEY.EDU \\ University of California, Berkeley \\ Department of Electrical Engineering and Computer Science \\ Berkeley, CA 94720 \\ Christian R. Shelton \\ CSHELTON@CS.UCR.EDU \\ University of California, Riverside \\ Department of Computer Science and Engineering \\ Riverside, CA 92521 \\ Daphne Koller \\ KOLLER@CS.STANFORD.EDU \\ Stanford University \\ Department of Computer Science \\ Stanford, CA 94305
}

\begin{abstract}
Structured game representations have recently attracted interest as models for multiagent artificial intelligence scenarios, with rational behavior most commonly characterized by Nash equilibria. This paper presents efficient, exact algorithms for computing Nash equilibria in structured game representations, including both graphical games and multi-agent influence diagrams (MAIDs). The algorithms are derived from a continuation method for normal-form and extensive-form games due to Govindan and Wilson; they follow a trajectory through a space of perturbed games and their equilibria, exploiting game structure through fast computation of the Jacobian of the payoff function. They are theoretically guaranteed to find at least one equilibrium of the game, and may find more. Our approach provides the first efficient algorithm for computing exact equilibria in graphical games with arbitrary topology, and the first algorithm to exploit fine-grained structural properties of MAIDs. Experimental results are presented demonstrating the effectiveness of the algorithms and comparing them to predecessors. The running time of the graphical game algorithm is similar to, and often better than, the running time of previous approximate algorithms. The algorithm for MAIDs can effectively solve games that are much larger than those solvable by previous methods.
\end{abstract}

\section{Introduction}

In attempting to reason about interactions between multiple agents, the artificial intelligence community has recently developed an interest in game theory, a tool from economics. Game theory is a very general mathematical formalism for the representation of complex multiagent scenarios, called games, in which agents choose actions and then receive payoffs that depend on the outcome of the game. A number of new game representations have been introduced in the past few years that exploit structure to represent games more efficiently. These representations are inspired by graphical models for probabilistic reasoning from the artificial intelligence literature, and include graphical games (Kearns, Littman, \& Singh, 
2001), multi-agent influence diagrams (MAIDs) (Koller \& Milch, 2001), G nets (La Mura, 2000), and action-graph games (Bhat \& Leyton-Brown, 2004).

Our goal is to describe rational behavior in a game. In game theory, a description of the behavior of all agents in the game is referred to as a strategy profile: a joint assignment of strategies to each agent. The most basic criterion to look for in a strategy profile is that it be optimal for each agent, taken individually: no agent should be able to improve its utility by changing its strategy. The fundamental game theoretic notion of a Nash equilibrium (Nash, 1951) satisfies this criterion precisely. A Nash equilibrium is a strategy profile in which no agent can improve its payoff by deviating unilaterally — changing its strategy while all other agents hold theirs fixed. There are other types of game theoretic solutions, but the Nash equilibrium is the most fundamental and is often agreed to be a minimum solution requirement.

Computing equilibria can be difficult for several reasons. First, game representations themselves can grow quite large. However, many of the games that we would be interested in solving do not require the full generality of description that leads to large representation size. The structured game representations introduced in AI exploit structural properties of games to represent them more compactly. Typically, this structure involves locality of interaction - agents are only concerned with the behavior of a subset of other agents.

One would hope that more compact representations might lead to more efficient computation of equilibria than would be possible with standard game-theoretic solution algorithms (such as those described by McKelvey \& McLennan, 1996). Unfortunately, even with compact representations, games are quite hard to solve; we present a result showing that finding Nash equilibria beyond a single trivial one is NP-hard in the types of structured games that we consider.

In this paper, we describe a set of algorithms for computing equilibria in structured games that perform quite well, empirically. Our algorithms are in the family of continuation methods. They begin with a solution of a trivial perturbed game, then track this solution as the perturbation is incrementally undone, following a trajectory through a space of equilibria of perturbed games until an equilibrium of the original game is found. Our algorithms are based on the recent work of Govindan and Wilson (2002, 2003, 2004) (GW hereafter), which applies to standard game representations (normal-form and extensive-form). The algorithms of GW are of great interest to the computational game theory community in their own right; Nudelman et al. (2004) have tested them against other leading algorithms and found them, in certain cases, to be the most effective available. However, as with all other algorithms for unstructured games, they are infeasible for very large games. We show how game structure can be exploited to perform the key computational step of the algorithms of GW, and also give an alternative presentation of their work.

Our methods address both graphical games and MAIDs. Several recent papers have presented methods for finding equilibria in graphical games. Many of the proposed algorithms (Kearns et al., 2001; Littman, Kearns, \& Singh, 2002; Vickrey \& Koller, 2002; Ortiz \& Kearns, 2003) have focused on finding approximate equilibria, in which each agent may in fact have a small incentive to deviate. These sorts of algorithms can be problematic: approximations must be crude for reasonable running times, and there is no guarantee of an exact equilibrium in the neighborhood of an approximate one. Algorithms that find exact equilibria have been restricted to a narrow class of games (Kearns et al., 2001). We 
present the first efficient algorithm for finding exact equilibria in graphical games of arbitrary structure. We present experimental results showing that the running time of our algorithm is similar to, and often better than, the running time of previous approximate algorithms. Moreover, our algorithm is capable of using approximate algorithms as starting points for finding exact equilibria.

The literature for MAIDs is more limited. The algorithm of Koller and Milch (2001) only takes advantage of certain coarse-grained structure in MAIDs, and otherwise falls back on generating and solving standard extensive-form games. Methods for related types of structured games (La Mura, 2000) are also limited to coarse-grained structure, and are currently unimplemented. Approximate approaches for MAIDs (Vickrey, 2002) come without implementation details or timing results. We provide the first exact algorithm that can take advantage of the fine-grained structure of MAIDs. We present experimental results demonstrating that our algorithm can solve MAIDs that are significantly outside the scope of previous methods.

\subsection{Outline and Guide to Background Material}

Our results require background in several distinct areas, including game theory, continuation methods, representations of graphical games, and representation and inference for Bayesian networks. Clearly, it is outside the scope of this paper to provide a detailed review of all of these topics. We have attempted to provide, for each of these topics, sufficient background to allow our results to be understood.

We begin with an overview of game theory in Section 2, describing strategy representations and payoffs in both normal-form games (single-move games) and extensive-form games (games with multiple moves through time). All concepts utilized in this paper will be presented in this section, but a more thorough treatment is available in the standard text by Fudenberg and Tirole (1991). In Section 3 we introduce the two structured game representations addressed in this paper: graphical games (derived from normal-form games) and MAIDs (derived from extensive-form games). In Section 4 we give a result on the complexity of computing equilibria in both graphical games and MAIDs, with the proof deferred to Appendix B. We next outline continuation methods, the general scheme our algorithms use to compute equilibria, in Section 5. Continuation methods form a broad computational framework, and our presentation is therefore necessarily limited in scope; Watson (2000) provides a more thorough grounding. In Section 6 we describe the particulars of applying continuation methods to normal-form games and to extensive-form games. The presentation is new, but the methods are exactly those of GW.

In Section 7, we present our main contribution: exploiting structure to perform the algorithms of GW efficiently on both graphical games and MAIDs. We show how Bayesian network inference in MAIDs can be used to perform the key computational step of the GW algorithm efficiently, taking advantage of finer-grained structure than previously possible. Our algorithm utilizes, as a subroutine, the clique tree inference algorithm for Bayesian networks. Although we do not present the clique tree method in full, we describe the properties of the method that allow it to be used within our algorithm; we also provide enough detail to allow an implementation of our algorithm using a standard clique tree package as a black box. For a more comprehensive introduction to inference in Bayesian 
networks, we refer the reader to the reference by Cowell, Dawid, Lauritzen, and Spiegelhalter (1999). In Section 8, we present running-time results for a variety of graphical games and MAIDs. We conclude in Section 9.

\section{Game Theory}

We begin by briefly reviewing concepts from game theory used in this paper, referring to the text by Fudenberg and Tirole (1991) for a good introduction. We use the notation employed by GW. Those readers more familiar with game theory may wish to skip directly to the table of notation in Appendix A.

A game defines an interaction between a set $N=\left\{n_{1}, n_{2}, \ldots, n_{|N|}\right\}$ of agents. Each agent $n \in N$ has a set $\Sigma_{n}$ of available strategies, where a strategy determines the agent's behavior in the game. The precise definition of the set $\Sigma_{n}$ depends on the game representation, as we discuss below. A strategy profile $\sigma=\left(\sigma_{n_{1}}, \sigma_{n_{2}}, \ldots, \sigma_{n_{|N|}}\right) \in \prod_{n \in N} \Sigma_{n}$ defines a strategy $\sigma_{n} \in \Sigma_{n}$ for each agent $n \in N$. Given a strategy profile $\sigma$, the game defines an expected payoff $G_{n}(\sigma)$ for each agent $n \in N$. We use $\Sigma_{-n}$ to refer to the set of all strategy profiles of agents in $N \backslash\{n\}$ (agents other than $n$ ) and $\sigma_{-n} \in \Sigma_{-n}$ to refer to one such profile; we generalize this notation to $\Sigma_{-n, n^{\prime}}$ for the set of strategy profiles of all but two agents. If $\sigma$ is a strategy profile, and $\sigma_{n}^{\prime} \in \Sigma_{n}$ is a strategy for agent $n$, then $\left(\sigma_{n}^{\prime}, \sigma_{-n}\right)$ is a new strategy profile in which $n$ deviates from $\sigma$ to play $\sigma_{n}^{\prime}$, and all other agents act according to $\sigma$.

A solution to a game is a prescription of a strategy profile for the agents. In this paper, we use Nash equilibria as our solution concept - strategy profiles in which no agent can profit by deviating unilaterally. If an agent knew that the others were playing according to an equilibrium profile (and would not change their behavior), it would have no incentive to deviate. Using the notation we have outlined here, we can define a Nash equilibrium to be a strategy profile $\sigma$ such that, for all $n \in N$ and all other strategies $\sigma_{n}^{\prime} \in \Sigma_{n}$, $G_{n}\left(\sigma_{n}, \sigma_{-n}\right) \geq G_{n}\left(\sigma_{n}^{\prime}, \sigma_{-n}\right)$.

We can also define a notion of an approximate equilibrium, in which each agent's incentive to deviate is small. An $\epsilon$-equilibrium is a strategy profile $\sigma$ such that no agent can improve its expected payoff by more than $\epsilon$ by unilaterally deviating from $\sigma$. In other words, for all $n \in N$ and all other strategies $\sigma_{n}^{\prime} \in \Sigma_{n}, G_{n}\left(\sigma_{n}^{\prime}, \sigma_{-n}\right)-G_{n}\left(\sigma_{n}, \sigma_{-n}\right) \leq \epsilon$. Unfortunately, finding an $\epsilon$-equilibrium is not necessarily a step toward finding an exact equilibrium: the fact that $\sigma$ is an $\epsilon$-equilibrium does not guarantee the existence of an exact equilibrium in the neighborhood of $\sigma$.

\subsection{Normal-Form Games}

A normal-form game defines a simultaneous-move multi-agent scenario. Each agent independently selects an action and then receives a payoff that depends on the actions selected by all of the agents. More precisely, let $G$ be a normal-form game with a set $N$ of agents. Each agent $n \in N$ has a discrete action set $A_{n}$ and a payoff array $G_{n}$ with entries for every action profile in $A=\prod_{n \in N} A_{n}$ - that is, for joint actions $\boldsymbol{a}=\left(a_{n_{1}}, a_{n_{2}}, \ldots, a_{n_{|N|}}\right)$ of all agents. We use $A_{-n}$ to refer to the joint actions of agents in $N \backslash\{n\}$. 


\subsubsection{Strategy Representation}

If agents are restricted to choosing actions deterministically, an equilibrium is not guaranteed to exist. If, however, agents are allowed to independently randomize over actions, then the seminal result of game theory (Nash, 1951) guarantees the existence of a mixed strategy equilibrium. A mixed strategy $\sigma_{n}$ is a probability distribution over $A_{n}$.

The strategy set $\Sigma_{n}$ is therefore defined to be the probability simplex of all mixed strategies. The support of a mixed strategy is the set of actions in $A_{n}$ that have non-zero probability. A strategy $\sigma_{n}$ for agent $n$ is said to be a pure strategy if it has only a single action in its support - pure strategies correspond exactly to the deterministic actions in $A_{n}$. The set $\Sigma$ of mixed strategy profiles is $\prod_{n \in N} \Sigma_{n}$, a product of simplices. A mixed strategy for a single agent can be represented as a vector of probabilities, one for each action. For notational simplicity later on, we can concatenate all these vectors and regard a mixed strategy profile $\sigma \in \Sigma$ as a single $m$-vector, where $m=\sum_{n \in N}\left|A_{n}\right|$. The vector is indexed by actions in $\cup_{n \in N} A_{n}$, so for an action $a \in A_{n}, \sigma_{a}$ is the probability that agent $n$ plays action $a$. (Note that, for notational convenience, every action is associated with a particular agent; different agents cannot take the "same" action.)

\subsubsection{PAYOFFS}

A mixed strategy profile induces a joint distribution over action profiles, and we can compute an expectation of payoffs with respect to this distribution. We let $G_{n}(\sigma)$ represent the expected payoff to agent $n$ when all agents behave according to the strategy profile $\sigma$. We can calculate this value by

$$
G_{n}(\sigma)=\sum_{\boldsymbol{a} \in A} G_{n}(\boldsymbol{a}) \prod_{k \in N} \sigma_{a_{k}} .
$$

In the most general case (a fully mixed strategy profile, in which every ), this sum includes every entry in the game array $G_{n}$, which is exponentially large in the number of agents.

\subsection{Extensive-Form Games}

An extensive-form game is represented by a tree. The game proceeds sequentially from the root. Each non-leaf node in the tree corresponds to a choice either of an agent or of nature; outgoing branches represent possible actions to be taken at the node. For each of nature's choice nodes, the game definition includes a probability distribution over the outgoing branches (these are points in the game at which something happens randomly in the world at large). Each leaf $z \in Z$ of the tree is an outcome, and is associated with a vector of payoffs $G(z)$, where $G_{n}(z)$ denotes the payoff to agent $n$ at leaf $z$. The choices of the agents and of nature dictate which path of the tree is followed.

The choice nodes belonging to each agent are partitioned into information sets; each information set is a set of states among which the agent cannot distinguish. Thus, an agent's strategy must dictate the same behavior at all nodes in the same information set. The set of agent $n$ 's information sets is denoted $I_{n}$, and the set of actions available at information set $i \in I_{n}$ is denoted $A(i)$. We define an agent history $H_{n}(y)$ for a node $y$ in the tree and an agent $n$ to be a sequence containing pairs $(i, a)$ of the information sets belonging to $n$ traversed in the path from the root to $y$ (excluding the information set in which $y$ itself is 


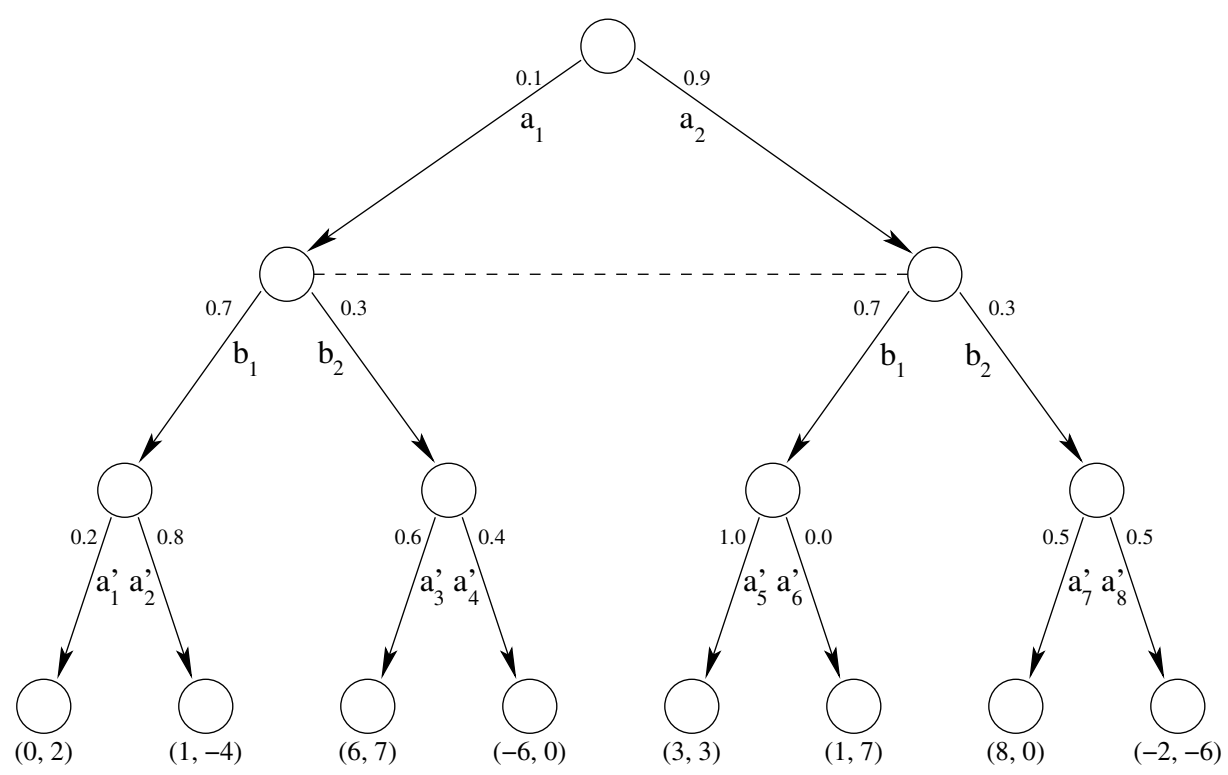

Figure 1: A simple 2-agent extensive-form game.

contained), and the action selected by $n$ at each one. Since actions are unique to information sets (the "same" action can't be taken at two different information sets), we can also omit the information sets and represent a history as an ordered tuple of actions only. Two nodes have the same agent- $n$ history if the paths used to reach them are indistinguishable to $n$, although the paths may differ in other ways, such as nature's decisions or the decisions of other agents. We make the common assumption of perfect recall: an agent does not forget information known nor choices made at its previous decisions. More precisely, if two nodes $y, y^{\prime}$ are in the same information set for agent $n$, then $H_{n}(y)=H_{n}\left(y^{\prime}\right)$.

Example 1. In the game tree shown in Figure 1, there are two agents, Alice and Bob. Alice first chooses between actions $a_{1}$ and $a_{2}$, Bob next chooses $b_{1}$ or $b_{2}$, and then Alice chooses between two of the set $\left\{a_{1}^{\prime}, a_{2}^{\prime}, \ldots, a_{8}^{\prime}\right\}$ (which pair depends on Bob's choice). Information sets are indicated by nodes connected with dashed lines. Bob is unaware of Alice's actions, so both of his nodes are in the same information set. Alice is aware at the bottom level of both her initial action and Bob's action, so each of her nodes is in a distinct information set. Edges have been labeled with the probability that the agent whose action it is will follow it; note that actions taken at nodes in the same information set must have the same probability distribution associated with them. There are eight possible outcomes of the game, each labeled with a pair of payoffs to Alice and Bob, respectively.

\subsubsection{Strategy Representation}

Unlike the case of normal-form games, there are several quite different choices of strategy representation for extensive-form games. One convenient formulation is in terms of behavior strategies. A behavior profile $b$ assigns to each information set $i$ a distribution over the 
actions $a \in A(i)$. The probability that agent $n$ takes action $a$ at information set $i \in I_{n}$ is then written $b(a \mid i)$. If $y$ is a node in $i$, then we can also write $b(a \mid y)$ as an abbreviation for $b(a \mid i)$.

Our methods primarily employ a variant of the sequence form representation (Koller \& Megiddo, 1992; von Stengel, 1996; Romanovskii, 1962), which is built upon the behavior strategy representation. In sequence form, a strategy $\sigma_{n}$ for an agent $n$ is represented as a realization plan, a vector of real values. Each value, or realization probability, in the realization plan corresponds to a distinct history (or sequence) $H_{n}(y)$ that agent $n$ has, over all nodes $y$ in the game tree. Some of these sequences may only be partial records of $n$ 's behavior in the game - proper prefixes of larger sequences. The strategy representation employed by GW (and by ourselves) is equivalent to the sequence form representation restricted to terminal sequences: those which are agent- $n$ histories of at least one leaf node. We shall henceforth refer to this modified strategy representation simply as "sequence form," for the sake of simplicity.

For agent $n$, then, we consider a realization plan $\sigma_{n}$ to be a vector of the realization probabilities of terminal sequences. For an outcome $z, \sigma\left(H_{n}(z)\right)$, abbreviated $\sigma_{n}(z)$, is the probability that agent $n$ 's choices allow the realization of outcome $z$ - in other words, the product of agent $n$ 's behavior probabilities along the history $H_{n}(z), \prod_{(i, a) \in H_{n}(z)} b(a \mid i)$. Several different outcomes may be associated with the same terminal sequence, so that agent $n$ may have fewer realization probabilities than there are leaves in the tree. The set of realization plans for agent $n$ is therefore a subset of $\mathbb{R}^{\ell_{n}}$, where $\ell_{n}$, the number of distinct terminal sequences for agent $n$, is at most the number of leaves in the tree.

Example 2. In the example above, Alice has eight terminal sequences, one for each of $a_{1}^{\prime}, a_{2}^{\prime}, \ldots, a_{8}^{\prime}$ from her four information sets at the bottom level. The history for one such last action is $\left(a_{1}, a_{3}^{\prime}\right)$. The realization probability $\sigma\left(a_{1}, a_{3}^{\prime}\right)$ is equal to $b\left(a_{1}\right) b\left(a_{3}^{\prime} \mid a_{1}, b_{2}\right)=$ $0.1 \cdot 0.6=0.06$. Bob has only two last actions, whose realization probabilities are exactly his behavior probabilities.

When all realization probabilities are non-zero, realization plans and behavior strategies are in one-to-one correspondence. (When some probabilities are zero, many possible behavior strategy profiles might correspond to the same realization plan, as described by Koller \& Megiddo, 1992; this does not affect the work presented here.) From a behavior strategy profile $b$, we can easily calculate the realization probability $\sigma_{n}(z)=\prod_{(i, a) \in H_{n}(z)} b(a \mid i)$. To understand the reverse transformation, note that we can also map behavior strategies to full realization plans defined on non-terminal sequences (as they were originally defined by Koller \& Megiddo, 1992) by defining $\sigma_{n}(h)=\prod_{(i, a) \in h} b(a \mid i)$; intuitively, $\sigma_{n}(h)$ is the probability that agent $n$ 's choices allow the realization of partial sequence $h$. Using this observation, we can compute a behavior strategy from an extended realization plan: if (partial) sequence $(h, a)$ extends sequence $h$ by one action, namely action $a$ at information set $i$ belonging to agent $n$, then we can compute $b(a \mid i)=\frac{\sigma_{n}(h, a)}{\sigma_{n}(h)}$. The extended realization probabilities can be computed from the terminal realization probabilities by a recursive procedure starting at the leaves of the tree and working upward: at information set $i$ with agent- $n$ history $h$ (determined uniquely by perfect recall), $\sigma_{n}(h)=\sum_{a \in A(i)} \sigma_{n}(h, a)$.

As several different information sets can have the same agent- $n$ history $h, \sigma_{n}(h)$ can be computed in multiple ways. In order for a (terminal) realization plan to be valid, it 
must satisfy the constraint that all choices of information sets with agent- $n$ history $h$ must give rise to the same value of $\sigma_{n}(h)$. More formally, for each partial sequence $h$, we have the constraints that for all pairs of information sets $i_{1}$ and $i_{2}$ with $H_{n}\left(i_{1}\right)=H_{n}\left(i_{2}\right)=h$, $\sum_{a \in A\left(i_{1}\right)} \sigma_{n}(h, a)=\sum_{a \in A\left(i_{2}\right)} \sigma_{n}(h, a)$. In the game tree of Example 1, consider Alice's realization probability $\sigma_{A}\left(a_{1}\right)$. It can be expressed as either $\sigma_{A}\left(a_{1}, a_{1}^{\prime}\right)+\sigma_{A}\left(a_{1}, a_{2}^{\prime}\right)=$ $0.1 \cdot 0.2+0.1 \cdot 0.8$ or $\sigma_{A}\left(a_{1}, a_{3}^{\prime}\right)+\sigma_{A}\left(a_{1}, a_{4}^{\prime}\right)=0.1 \cdot 0.6+0.1 \cdot 0.4$, so these two sums must be the same.

By recursively defining each realization probability as a sum of realization probabilities for longer sequences, all constraints can be expressed in terms of terminal realization probabilities; in fact, the constraints are linear in these probabilities. There are several further constraints: all probabilities must be nonnegative, and, for each agent $n, \sigma_{n}(\emptyset)=1$, where $\emptyset$ (the empty sequence) is the agent- $n$ history of the first information set that agent $n$ encounters. This latter constraint simply enforces that probabilities sum to one. Together, these linear constraints define a convex polytope $\Sigma$ of legal terminal realization plans.

\subsubsection{PAYOFFS}

If all agents play according to $\sigma \in \Sigma$, the payoff to agent $n$ in an extensive-form game is

$$
G_{n}(\sigma)=\sum_{z \in Z} G_{n}(z) \prod_{k \in N} \sigma_{k}(z)
$$

where here we have augmented $N$ to include nature for notational convenience. This is simply an expected sum of the payoffs over all leaves. For each agent $k, \sigma_{k}(z)$ is the product of the probabilities controlled by $n$ along the path to $z$; thus, $\prod_{k \in N} \sigma_{k}(z)$ is the multiplication of all probabilities along the path to $z$, which is precisely the probability of $z$ occurring. Importantly, this expression has a similar multi-linear form to the payoff in a normal-form game, using realization plans rather than mixed strategies.

Extensive-form games can be expressed (inefficiently) as normal-form games, so they too are guaranteed to have an equilibrium in mixed strategies. In an extensive-form game satisfying perfect recall, any mixed strategy profile can be represented by a payoff-equivalent behavior profile, and hence by a realization plan (Kuhn, 1953).

\section{Structured Game Representations}

The artificial intelligence community has recently introduced structured representations that exploit independence relations in games in order to represent them compactly. Our methods address two of these representations: graphical games (Kearns et al., 2001), a structured class of normal-form games, and MAIDs (Koller \& Milch, 2001), a structured class of extensive-form games.

\subsection{Graphical Games}

The size of the payoff arrays required to describe a normal-form game grows exponentially with the number of agents. In order to avoid this blow-up, Kearns et al. (2001) introduced the framework of graphical games, a more structured representation inspired by probabilistic graphical models. Graphical games capture local structure in multi-agent interactions, 
allowing a compact representation for scenarios in which each agent's payoff is only affected by a small subset of other agents. Examples of interactions where this structure occurs include agents that interact along organization hierarchies and agents that interact according to geographic proximity.

A graphical game is similar in definition to a normal-form game, but the representation is augmented by the inclusion of an interaction graph with a node for each agent. The original definition assumed an undirected graph, but easily generalizes to directed graphs. An edge from agent $n^{\prime}$ to agent $n$ in the graph indicates that agent $n$ 's payoffs depend on the action of agent $n^{\prime}$. More precisely, we define $\mathrm{Fam}_{n}$ to be the set of agents consisting of $n$ itself and its parents in the graph. Agent $n$ 's payoff function $G_{n}$ is an array indexed only by the actions of the agents in $\mathrm{Fam}_{n}$. Thus, the description of the game is exponential in the in-degree of the graph and not in the total number of agents. In this case, we use

$\Sigma_{-n}^{f}$ and $A_{-n}^{f}$ to refer to strategy profiles and action profiles, respectively, of the agents in Fam $_{n} \backslash\{n\}$.

Example 3. Suppose $2 L$ landowners along a road running north to south are deciding whether to build a factory, a residential neighborhood, or a shopping mall on their plots. The plots are laid out along the road in a 2-by-L grid; half of the agents are on the east side $\left(e_{1}, \ldots, e_{L}\right)$ and half are on the west side $\left(w_{1}, \ldots, w_{L}\right)$. Each agent's payoff depends only on what it builds and what its neighbors to the north, south, and across the road build. For example, no agent wants to build a residential neighborhood next to a factory. Each agent's payoff matrix is indexed by the actions of at most four agents (fewer at the ends of the road) and has $3^{4}$ entries, as opposed to the full $3^{2 L}$ entries required in the equivalent normal form game. (This example is due to Vickrey 8 Koller, 2002.)

\subsection{Multi-Agent Influence Diagrams}

The description length of extensive-form games can also grow exponentially with the number of agents. In many situations, this large tree can be represented more compactly. Multi-agent influence diagrams (MAIDs) (Koller \& Milch, 2001) allow a structured representation of games involving time and information by extending influence diagrams (Howard \& Matheson, 1984) to the multi-agent case.

MAIDs and influence diagrams derive much of their syntax and semantics from the Bayesian network framework. A MAID compactly represents a certain type of extensiveform game in much the same way that a Bayesian network compactly represents a joint probability distribution. For a thorough treatment of Bayesian networks, we refer the reader to the reference by Cowell et al. (1999).

\subsubsection{MAID REPRESENTATION}

Like a Bayesian network, a MAID defines a directed acyclic graph whose nodes correspond to random variables. These random variables are partitioned into sets: a set $\mathcal{X}$ of chance variables whose values are chosen by nature, represented in the graph by ovals; for each agent $n$, a set $\mathcal{D}_{n}$ of decision variables whose values are chosen by agent $n$, represented by rectangles; and for each agent $n$, a set $\mathcal{U}_{n}$ of utility variables, represented by diamonds. Chance and decision variables have, as their domains, finite sets of possible actions. We refer to the domain of a random variable $V$ by $\operatorname{dom}(V)$. For each chance or decision variable 
$V$, the graph defines a parent set $P a_{V}$ of those variables on whose values the choice at $V$ can depend. Utility variables have finite sets of real payoff values for their domains, and are not permitted to have children in the graph; they represent components of an agent's payoffs, and not game state.

The game definition supplies each chance variable $X$ with a conditional probability distribution (CPD) $P\left(X \mid P a_{X}\right)$, conditioned on the values of the parent variables of $X$. The semantics for a chance variable are identical to the semantics of a random variable in a Bayesian network; the CPD specifies the probability that an action in $\operatorname{dom}(X)$ will be selected by nature, given the actions taken at $X$ 's parents. The game definition also supplies a utility function for each utility node $U$. The utility function maps each instantiation $\boldsymbol{p a} \in \operatorname{dom}\left(P a_{U}\right)$ deterministically to a real value $U(\boldsymbol{p a})$. For notational and algorithmic convenience, we can regard this utility function as a CPD $P\left(U \mid P a_{U}\right)$ in which, for each $\boldsymbol{p a} \in \operatorname{dom}\left(P a_{U}\right)$, the value $U(\boldsymbol{p a})$ has probability 1 in $P(U \mid \boldsymbol{p a})$ and all other values have probability 0 (the domain of $U$ is simply the finite set of possible utility values). At the end of the game, agent $n$ 's total payoff is the sum of the utility received from each $U_{n}^{i} \in \mathcal{U}_{n}$ (here $i$ is an index variable). Note that each component $U_{n}^{i}$ of agent $n$ 's payoff depends only on a subset of the variables in the MAID; the idea is to compactly decompose $a$ 's payoff into additive pieces.

\subsubsection{Strategy Representation}

The counterpart of a CPD for a decision node is a decision rule. A decision rule for a decision variable $D_{n}^{i} \in \mathcal{D}_{n}$ is a function, specified by $n$, mapping each instantiation $\boldsymbol{p a} \in \operatorname{dom}\left(P a_{D_{n}^{i}}\right)$ to a probability distribution over the possible actions in $\operatorname{dom}\left(D_{n}^{i}\right)$. A decision rule is identical in form to a conditional probability distribution, and we can refer to it using the notation $P\left(D_{n}^{i} \mid P a_{D_{n}^{i}}\right)$. As with the semantics for a chance node, the decision rule specifies the probability that agent $n$ will take any particular action in $\operatorname{dom}\left(D_{n}^{i}\right)$, having seen the actions taken at $D_{n}^{i}$ 's parents. An assignment of decision rules to all $D_{n}^{i} \in \mathcal{D}_{n}$ comprises a strategy for agent $n$. Once agent $n$ chooses a strategy, $n$ 's behavior at $D_{n}^{i}$ depends only on the actions taken at $D_{n}^{i}$ 's parents. $P a_{D_{n}^{i}}$ can therefore be regarded as the set of nodes whose values are visible to $n$ when it makes its choice at $D_{n}^{i}$. Agent $n$ 's choice of strategy may well take other nodes into account; but during actual game play, all nodes except those in $P a_{D_{n}^{i}}$ are invisible to $n$.

Example 4. The extensive-form game considered in Example 1 can be represented by the MAID shown in Figure 2(a). Alice and Bob each have an initial decision to make without any information about previous actions; then Alice has another decision to make in which she is aware of Bob's action and her own. Alice and Bob each have only one utility node (the two are condensed into a single node in the graph, for the sake of brevity), whose payoff structure is wholly general (dependent on every action in the game) and thus whose possible values are exactly the values from the payoff vectors in the extensive-form game.

Example 5. Figure 2(b) shows a more complicated MAID of a somewhat more realistic scenario. Here, three landowners along a road are deciding whether to build a store or a house. Their payoff depends only on what happens adjacent to them along the road. Their decision proceeds in two stages: the planning stage and the building stage. The second 


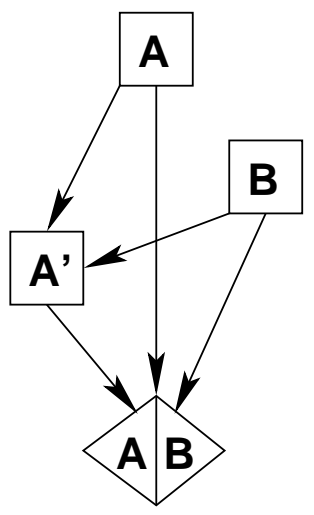

(a)

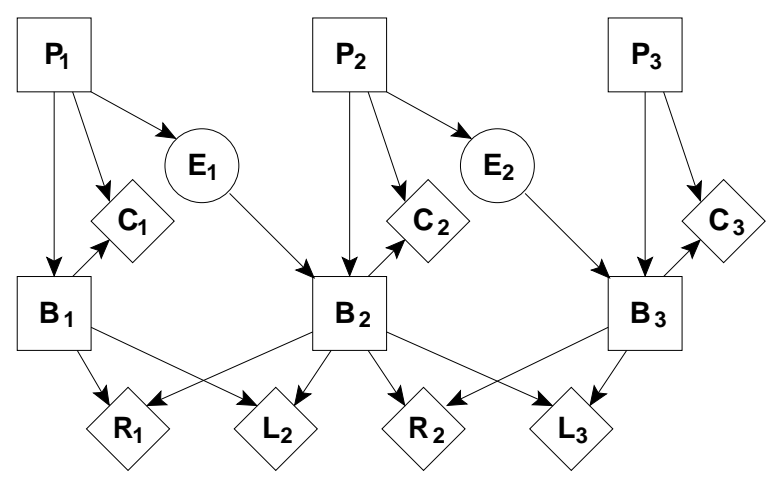

(b)

Figure 2: (a) A simple MAID equivalent to the extensive form game in Figure 1. (b) A two-stage road game with three agents.

landowner, for instance, has the two decision variables $P_{2}$ and $B_{2}$. He receives a certain penalty from the utility node $C_{2}$ if he builds the opposite of what he had planned to build. But after planning, he learns something about what his neighbor to the left has planned. The chance node $E_{1}$ represents noisy espionage; it transmits the action taken at $P_{1}$. After learning the value of $E_{1}$, it may be in the second landowner's interests to deviate from his plan, even if it means incurring the penalty. It is in his interest to start a trend that distinguishes him from previous builders but which subsequent builders will follow: the utility node $L_{2}$ rewards him for building the opposite of what was built at $B_{1}$, and the utility node $R_{2}$ rewards him if the third landowner builds the same thing he does at $B_{3}$.

Note that this MAID exhibits perfect recall, because the choice made at a planning stage is visible to the agent when it makes its next choice at the building stage.

\subsubsection{PAYOFFS}

Under a particular strategy profile $\sigma$ - that is, a tuple of strategies for all players - all decision nodes have CPDs specified. Since chance and utility nodes are endowed with CPDs already, the MAID therefore induces a fully-specified Bayesian network $\mathcal{B}_{\sigma}$ with variables $\mathcal{V}=\mathcal{X} \cup \mathcal{D} \cup \mathcal{U}$ and the same directed graph as the MAID. By the chain rule for Bayesian networks, $\mathcal{B}_{\sigma}$ induces a joint probability distribution $P_{\sigma}$ over all the variables in $\mathcal{V}$ by $P_{\sigma}(\mathcal{V})=\prod_{V \in \mathcal{V}} P\left(V \mid P a_{V}\right)$, with CPDs for chance and utility variables given by the MAID definition and CPDs for decision variables given by $\sigma$. For a game $G$ represented as a MAID, the expected payoff that agent $n$ receives under $\sigma$ is the expectation of $n$ 's utility node values with respect to this distribution:

$$
\begin{aligned}
G_{n}(\sigma) & =\sum_{U_{n}^{i} \in \mathcal{U}_{n}} E_{P_{\sigma}}\left[U_{n}^{i}\right] \\
& =\sum_{U_{n}^{i} \in \mathcal{U}_{n}} \sum_{u \in \operatorname{dom}\left(U_{n}^{i}\right)} u \cdot P_{\sigma}(u) .
\end{aligned}
$$


We show in Section 7 that this and other related expectations can be calculated efficiently using Bayesian network inference algorithms, giving a substantial performance increase over the calculation of payoffs in the extensive-form game.

\subsubsection{Extensive Form Strategy Representations in MAiDs}

A MAID provides a compact definition of an extensive-form game. We note that, although this correspondence between MAIDs and extensive form games provides some intuition about MAIDs, the details of the mapping are not relevant to the remainder of the discussion. We therefore briefly review this construction, referring to the work of Koller and Milch (2001) for details.

The game tree associated with a MAID is a full, balanced tree, with each path corresponding to a complete assignment of the chance and decision nodes in the network. Each node in the tree corresponds either to a chance node or to a decision node of one of the players, with an outgoing branch for each possible action at that node. All nodes at the same depth in the tree correspond to the same MAID node. We assume that the nodes along a path in the tree are ordered consistently with the ordering implied by the directed edges in the MAID, so that if a MAID node $X$ is a parent of a MAID node $Y$, the tree branches on $X$ before it branches on $Y$. The information sets for tree nodes associated with a decision node $D_{n}^{i}$ correspond to assignments to the parents $P a_{D_{n}^{i}}$ : all tree nodes corresponding to $D_{n}^{i}$ with the same assignment to $P a_{D_{n}^{i}}$ are in a single information set. We note that, by construction, the assignment to $P a_{D_{n}^{i}}$ was determined earlier in the tree, and so the partition to information sets is well-defined. For example, the simple MAID in Figure 2(a) expands into the much larger game tree that we saw earlier in Figure 1.

Translating in the opposite direction, from extensive-form games to MAIDs, is not always as natural. If the game tree is unbalanced, then we cannot simply reverse the above process. However, with care, it is possible to construct a MAID that is no larger than a given extensive-form game, and that may be exponentially smaller in the number of agents. The details are fairly technical, and we omit them here in the interest of brevity.

Despite the fact that a MAID will typically be much more compact than the equivalent extensive-form game, the strategy representations of the two turn out to be equivalent and of equal size. A decision rule for a decision variable $D_{n}^{i}$ assigns a distribution over actions to each joint assignment to $P a_{D_{n}^{i}}$, just as a behavior strategy assigns a distribution over actions to an information set in an extensive form game - as discussed above, each assignment to the parents of $D_{n}^{i}$ is an information set. A strategy profile for a MAID - a set of decision rules for every decision variable - is therefore equivalent to a set of behavior strategies for every information set, which is simply a behavior profile.

If we make the assumption of perfect recall, then, since MAID strategies are simply behavior strategies, we can represent them in sequence form. Perfect recall requires that no agent forget anything that it has learned over the course of the game. In the MAID formalism, the perfect recall assumption is equivalent to the following constraint: if agent $n$ has two decision nodes $D_{n}^{i}$ and $D_{n}^{j}$, with the second occurring after the first, then all parents of $D_{n}^{i}$ (the information $n$ is aware of in making decision $D_{n}^{i}$ ) and $D_{n}^{i}$ itself must be parents of $D_{n}^{j}$. This implies that agent $n$ 's final decision node $D_{n}^{d}$ has, as parents, all of $n$ 's previous decision nodes and their parents. Then a joint assignment to $D_{n}^{d} \cup P a_{D_{n}^{d}}$ precisely 
determines agent $n$ 's sequence of information sets and actions leading to an outcome of the game - the agent- $n$ history of the outcome.

The realization probability for a particular sequence is computed by multiplying all behavior strategy probabilities for actions in that sequence. In MAIDs, a sequence cor-

responds to a joint assignment to $D_{n}^{d} \cup P a_{D_{n}^{d}}$, and the behavior strategy probabilities for this sequence are entries consistent with this assignment in the decision rules for agent $n$. We can therefore derive all of agent $n$ 's realization probabilities at once by multiplying together, as conditional probability distributions, the decision rules of each of agent $n$ 's decision nodes in the sequence - when multiplying conditional probability distributions, only those entries whose assignments are consistent with each other are multiplied. Conversely, given a realization plan, we can derive the behavior strategies and hence the decision rules according to the method outlined for extensive-form games.

In the simple MAID example in Figure 2(a), the terminal sequences are the same as in the equivalent extensive-form game. In the road example in Figure 2(b), agent 2 has 8 terminal sequences; one for each joint assignment to his final decision node $\left(B_{2}\right)$ and its parents $\left(E_{1}\right.$ and $\left.P_{2}\right)$. Their associated realization probabilities are given by multiplying the decision rules at $P_{2}$ and at $B_{2}$.

\section{Computational Complexity}

When developing algorithms to compute equilibria efficiently, the question naturally arises of how well one can expect these algorithms to perform. The complexity of computing Nash equilibria has been studied for some time. Gilboa and Zemel (1989) first showed that it is NP-hard to find more than one Nash equilibrium in a normal-form game, and Conitzer and Sandholm (2003) recently utilized a simpler reduction to arrive at this result and several others in the same vein. Other recent hardness results pertain to restricted subclasses of normal-form games (e.g., Chu \& Halpern, 2001; Codenotti \& Stefankovic, 2005). However, these results apply only to 2-agent normal-form games. While it is true that proving a certain subclass of a class of problems to be NP-hard also proves the entire class to be NP-hard (because NP-hardness is a measure of worst-case complexity), such a proof might tell us very little about the complexity of problems outside the subclass. This issue is particularly apparent in the problem of computing equilibria, because games can grow along two distinct axes: the number of agents, and the number of actions per agent. The hardness results of Conitzer and Sandholm (2003) apply only as the number of actions per agent increases. Because 2-agent normal-form games are (fully connected) graphical games, these results apply to graphical games.

However, we are more interested in the hardness of graphical games as the number of agents increases, rather than the number of actions per agent. It is graphical games with large numbers of agents that capture the most structure - these are the games for which the graphical game representation was designed. In order to prove results about the asymptotic hardness of computing equilibria along this more interesting (in this setting) axis of representation size, we require a different reduction. Our proof, like a number of previous hardness proofs for games (e.g., Chu \& Halpern, 2001; Conitzer \& Sandholm, 2003; Codenotti \& Stefankovic, 2005), reduces 3SAT to equilibrium computation. However, in these previous proofs, variables in 3SAT instances are mapped to actions (or sets of actions) 
in a game with only 2 players, whereas in our reduction they are mapped to agents. Although differing in approach, our reduction is very much in the spirit of the reduction appearing in the work of Conitzer and Sandholm (2003), and many of the corollaries of their main result also follow from ours (in a form adapted to graphical games).

Theorem 6. For any constant $d \geq 5$, and $k \geq 2$, the problem of deciding whether $a$ graphical game with a family size at most $d$ and at most $k$ actions per player has more than one Nash equilibrium is NP-hard.

Proof. Deferred to Appendix B.

In our reduction, all games that have more than one equilibria have at least one pure strategy equilibrium. This immediately gives us

Corollary 7. It is NP-hard to determine whether a graphical game has more than one Nash equilibrium in discretized strategies with even the coarsest possible granularity.

Finally, because graphical games can be represented as (trivial) MAIDs, in which each agent has only a single parentless decision node and a single utility node, and each agent's utility node has, as parents, the decision nodes of the graphical game family of that agent, we obtain the following corollary.

Corollary 8. It is NP-hard to determine whether a MAID with constant family size at least 6 has more than one Nash equilibrium.

\section{Continuation Methods}

Continuation methods form the basis of our algorithms for solving each of these structured game representations. We begin with a high-level overview of continuation methods, referring the reader to the work of Watson (2000) for a more detailed discussion.

Continuation methods work by solving a simpler perturbed problem and then tracing the solution as the magnitude of the perturbation decreases, converging to a solution for the original problem. More precisely, let $\lambda$ be a scalar parameterizing a continuum of perturbed problems. When $\lambda=0$, the perturbed problem is the original one; when $\lambda=1$, the perturbed problem is one for which the solution is known. Let $\boldsymbol{w}$ represent the vector of real values of the solution. For any perturbed problem defined by $\lambda$, we characterize solutions by the equation $F(\boldsymbol{w}, \lambda)=\mathbf{0}$, where $F$ is a real-valued vector function of the same dimension as $\boldsymbol{w}$ (so that $\mathbf{0}$ is a vector of zeros). The function $F$ is such that $\boldsymbol{w}$ is a solution to the problem perturbed by $\lambda$ if and only if $F(\boldsymbol{w}, \lambda)=\mathbf{0}$.

The continuation method traces solutions along the level set of solution pairs $(\boldsymbol{w}, \lambda)$ satisfying $F(\boldsymbol{w}, \lambda)=\mathbf{0}$. Specifically, if we have a solution pair $(\boldsymbol{w}, \lambda)$, we would like to trace that solution to a nearby solution. Differential changes to $\boldsymbol{w}$ and $\lambda$ must cancel out so that $F$ remains equal to $\mathbf{0}$.

If $(\boldsymbol{w}, \lambda)$ changes in the direction of a unit vector $\boldsymbol{u}$, then $F$ will change in the direction $\nabla F \cdot \boldsymbol{u}$, where $\nabla F$ is the Jacobian of $F$ (which can also be written $\left[\begin{array}{lll}\nabla_{\boldsymbol{w}} F & \nabla_{\lambda} F\end{array}\right]$ ). We want to find a direction $\boldsymbol{u}$ such that $F$ remains unchanged, i.e., equal to $\mathbf{0}$. Thus, we need to solve the matrix equation

$$
\left[\begin{array}{ll}
\nabla_{\boldsymbol{w}} F & \nabla_{\lambda} F
\end{array}\right]\left[\begin{array}{c}
d \boldsymbol{w} \\
d \lambda
\end{array}\right]=0
$$


Equivalently, changes $d \boldsymbol{w}$ and $d \lambda$ along the path must obey $\nabla_{\boldsymbol{w}} F \cdot d \boldsymbol{w}=-\nabla_{\lambda} F \cdot d \lambda$. Rather than inverting the matrix $\nabla_{\boldsymbol{w}} F$ in solving this equation, we use the adjoint $\operatorname{adj}\left(\nabla_{\boldsymbol{w}} F\right)$, which is still defined when $\nabla_{\boldsymbol{w}} F$ has a null space of rank 1. The adjoint is the matrix of cofactors: the element at $(i, j)$ is $(-1)^{i+j}$ times the determinant of the sub-matrix in which row $i$ and column $j$ have been removed. When the inverse is defined, $\operatorname{adj}\left(\nabla_{\boldsymbol{w}} F\right)=\operatorname{det}\left(\nabla_{\boldsymbol{w}} F\right)\left[\nabla_{\boldsymbol{w}} F\right]^{-1}$. In practice, we therefore set $d \boldsymbol{w}=-\operatorname{adj}\left(\nabla_{\boldsymbol{w}} F\right) \cdot \nabla_{\lambda} F$ and $d \lambda=\operatorname{det}\left(\nabla_{\boldsymbol{w}} F\right)$. If the Jacobian $\left[\begin{array}{lll}\nabla_{\boldsymbol{w}} F & \nabla_{\lambda} F\end{array}\right]$ has a null-space of rank 1 everywhere, the curve is uniquely defined.

The function $F$ should be constructed so that the curve starting at $\lambda=1$ is guaranteed to cross $\lambda=0$, at which point the corresponding value of $\boldsymbol{w}$ is a solution to the original problem. A continuation method begins at the known solution for $\lambda=1$. The null-space of the Jacobian $\nabla F$ at a current solution $(\boldsymbol{w}, \lambda)$ defines a direction, along which the solution is moved by a small amount. The Jacobian is then recalculated and the process repeats, tracing the curve until $\lambda=0$. The cost of each step in this computation is at least cubic in the size of $\boldsymbol{w}$, due to the required matrix operations. However, the Jacobian itself may in general be much more difficult to compute. Watson (2000) provides some simple examples of continuation methods.

\section{Continuation Methods for Games}

We now review the work of GW on applying the continuation method to the task of finding equilibria in games. They provide continuation methods for both normal-form and extensive-form games. These algorithms form the basis for our extension to structured games, described in the next section. The continuation methods perturb the game by giving agents fixed bonuses, scaled by $\lambda$, for each of their actions, independently of whatever else happens in the game. If the bonuses are large enough (and unique), they dominate the original game structure, and the agents need not consider their opponents' actions. There is thus a unique pure-strategy equilibrium easily determined by the bonuses at $\lambda=1$. The continuation method can then be used to follow a path in the space of $\lambda$ and equilibrium profiles for the resulting perturbed game, decreasing $\lambda$ until it is zero; at this point, the corresponding strategy profile is an equilibrium of the original game.

\subsection{Continuation Method for Normal-Form Games}

We now make this intuition more precise, beginning with normal-form games.

\subsubsection{Perturbations}

A perturbation vector $\boldsymbol{b}$ is a vector of $m$ values chosen at random, one for each action in the game. The bonus $b_{a}$ is given to the agent $n$ owning action $a$ for playing $a$, independently of whatever else happens in the game. Applying this perturbation to a target game $G$ gives us a new game, which we denote $G \oplus \boldsymbol{b}$, in which, for each $a \in A_{n}$, and for any $\boldsymbol{t} \in A_{-n}$, $(G \oplus \boldsymbol{b})_{n}(a, \boldsymbol{t})=G_{n}(a, \boldsymbol{t})+b_{a}$. If $\boldsymbol{b}$ is made sufficiently large, then $G \oplus \boldsymbol{b}$ has a unique equilibrium, in which each agent plays the pure strategy $a$ for which $b_{a}$ is maximal. 


\subsubsection{Characterization of Equilibria}

In order to apply Equation (3), we need to characterize the equilibria of perturbed games as the zeros of a function $F$. Using a structure theorem of Kohlberg and Mertens (1986), GW show that the continuation method path deriving from their equilibrium characterization leads to convergence for all perturbation vectors except those in a set of measure zero. We present only the equilibrium characterization here; proofs of the characterization and of the method's convergence are given by Govindan and Wilson (2003).

We first define an auxiliary vector function $V^{G}(\sigma)$, indexed by actions, of the payoffs to each agent for deviating from $\sigma$ to play a single action. We call $V^{G}$ the deviation function. The element $V_{a}^{G}(\sigma)$ corresponding to a single action $a$, owned by an agent $n$, is the payoff to agent $n$ when it deviates from the mixed strategy profile $\sigma$ by playing the pure strategy for action $a$ :

$$
V_{a}^{G}(\sigma)=\sum_{\boldsymbol{t} \in A_{-n}} G_{n}(a, \boldsymbol{t}) \prod_{k \in N \backslash\{n\}} \sigma_{t_{k}} .
$$

It can also be viewed as the component of agent $n$ 's payoff that it derives from action $a$, under the strategy profile $\sigma$. Since bonuses are given to actions independently of $\sigma$, the effect of bonuses on $V^{G}$ is independent of $\sigma$. $V_{a}^{G}$ measures the payoff for deviating and playing $a$, and bonuses are given for precisely this deviation, so $V^{G \oplus \boldsymbol{b}}(\sigma)=V^{G}(\sigma)+\boldsymbol{b}$.

We also utilize the retraction operator $R: \mathbb{R}^{m} \rightarrow \Sigma$ defined by Gül, Pearce, and Stachetti (1993), which maps an arbitrary $m$-vector $\boldsymbol{w}$ to the point in the space $\Sigma$ of mixed strategies which is nearest to $\boldsymbol{w}$ in Euclidean distance. Given this operator, the equilibrium characterization is as follows.

Lemma 9. (Gül et al., 1993) If $\sigma$ is a strategy profile of $G$, then $\sigma=R\left(V^{G}(\sigma)+\sigma\right)$ iff $\sigma$ is an equilibrium.

Although we omit the proof, we will give some intuition for why this result is true. Suppose $\sigma$ is a fully-mixed equilibrium; that is, every action has non-zero probability. For a single agent $n, V_{a}^{G}(\sigma)$ must be the same for all actions $a \in A_{n}$, because $n$ should not have any incentive to deviate and play a single one of them. Let $V_{n}$ be the vector of entries in $V^{G}(\sigma)$ corresponding to actions of $n$, and let $\sigma_{n}$ be defined similarly. $V_{n}$ is a scalar multiple of $\mathbf{1}$, the all-ones vector, and the simplex $\Sigma_{n}$ of $n$ 's mixed strategies is defined by $\mathbf{1}^{T} \boldsymbol{x}=1$, so $V_{n}$ is orthogonal to $\Sigma_{n}$. $V^{G}(\sigma)$ is therefore orthogonal to $\Sigma$, so retracting $\sigma+V^{G}(\sigma)$ onto $\Sigma$ gives precisely $\sigma$. In the reverse direction, if $\sigma$ is a fully-mixed strategy profile satisfying $\sigma=R\left(V^{G}(\sigma)+\sigma\right)$, then $V^{G}(\sigma)$ must be orthogonal to the polytope of mixed strategies. Then, for each agent, every pure strategy has the same payoff. Therefore, $\sigma$ is in fact an equilibrium. A little more care must be taken when dealing with actions not in the support. We refer to Gül et al. (1993) for the details.

According to Lemma 9, we can define an equilibrium as a solution to the equation $\sigma=R\left(\sigma+V^{G}(\sigma)\right)$. On the other hand, if $\sigma=R(\boldsymbol{w})$ for some $\boldsymbol{w} \in \mathbb{R}^{m}$, we have the equivalent condition that $\boldsymbol{w}=R(\boldsymbol{w})+V^{G}(R(\boldsymbol{w})) ; \sigma$ is an equilibrium iff this condition is satisfied, as can easily be verified. We can therefore search for a point $\boldsymbol{w} \in \mathbb{R}^{m}$ which satisfies this equality, in which case $R(\boldsymbol{w})$ is guaranteed to be an equilibrium.

The form of our continuation equation is then

$$
F(\boldsymbol{w}, \lambda)=\boldsymbol{w}-R(\boldsymbol{w})-\left(V^{G}(R(\boldsymbol{w}))+\lambda \boldsymbol{b}\right) .
$$


We have that $V^{G}+\lambda \boldsymbol{b}$ is the deviation function for the perturbed game $G \oplus \lambda \boldsymbol{b}$, so $F(\boldsymbol{w}, \lambda)$ is zero if and only if $R(\boldsymbol{w})$ is an equilibrium of $G \oplus \lambda \boldsymbol{b}$. At $\lambda=0$ the game is unperturbed, so $F(\boldsymbol{w}, 0)=\mathbf{0}$ iff $R(\boldsymbol{w})$ is an equilibrium of $G$.

\subsubsection{Computation}

The expensive step in the continuation method is the calculation of the Jacobian $\nabla_{\boldsymbol{w}} F$, required for the computation that maintains the constraint of Equation (3). Here, we have that $\nabla_{\boldsymbol{w}} F=I-\left(I+\nabla V^{G}\right) \nabla R$, where $I$ is the $m \times m$ identity matrix. The hard part is the calculation of $\nabla V^{G}$. For pure strategies $a \in A_{n}$ and $a^{\prime} \in A_{n^{\prime}}$, for $n^{\prime} \neq n$, the value at location $\left(a, a^{\prime}\right)$ in $\nabla V^{G}(\sigma)$ is equal to the expected payoff to agent $n$ when it plays the pure strategy $a$, agent $n^{\prime}$ plays the pure strategy $a^{\prime}$, and all other agents act according to the strategy profile $\sigma$ :

$$
\begin{aligned}
\nabla V_{a, a^{\prime}}^{G}(\sigma) & =\frac{\partial}{\partial \sigma_{a^{\prime}}} \sum_{\boldsymbol{t} \in A_{-n}} G_{n}(a, \boldsymbol{t}) \prod_{k \in N \backslash\{n\}} \sigma_{\boldsymbol{t}_{k}} \\
& =\sum_{\boldsymbol{t} \in A_{-n, n^{\prime}}} G_{n}\left(a, a^{\prime}, \boldsymbol{t}\right) \prod_{k \in N \backslash\left\{n, n^{\prime}\right\}} \sigma_{\boldsymbol{t}_{k}}
\end{aligned}
$$

If both $a \in A_{n}$ and $a^{\prime} \in A_{n}, \nabla V_{a, a^{\prime}}^{G}(\sigma)=0$.

Computing Equation (6) requires a large number of multiplications; the sum is over the space $A_{-n, n^{\prime}}=\prod_{k \in N \backslash\left\{n, n^{\prime}\right\}} A_{i}$, which is exponentially large in the number of agents.

\subsection{Continuation Method for Extensive-Form Games}

The same method applies to extensive-form games, using the sequence form strategy representation.

\subsubsection{Perturbations}

As with normal-form games, the game is perturbed by the bonus vector $\boldsymbol{b}$. Agent $n$ owning sequence $h$ is paid an additional bonus $\boldsymbol{b}_{h}$ for playing $h$, independently of whatever else happens in the game. Applying this perturbation gives us a new game $G \oplus \boldsymbol{b}$ in which, for each $z \in Z,(G \oplus \boldsymbol{b})_{n}(z)=G_{n}(z)+\boldsymbol{b}_{H_{n}(z)}$.

If the bonuses are large enough and unique, GW show that once again the perturbed game has a unique pure-strategy equilibrium (one in which all realization probabilities are 0 or 1). However, calculating it is not as simple as in the case of normal-form games. Behavior strategies must be calculated from the leaves upward by a recursive procedure, in which at each step the agent who owns the node in question chooses the action that results in the sequence with the largest bonus. Since all actions below it have been recursively determined, each action at the node in question determines an outcome. The realization plans can be derived from this behavior profile by the method outlined in Section 2.2.1. 


\subsubsection{Characterization of Equilibria}

Once more, we first define a vector function capturing the benefit of deviating from a given strategy profile, indexed by sequences:

$$
V_{h}^{G}(\sigma)=\sum_{z \in Z_{h}} G_{n}(z) \prod_{k \in N \backslash\{n\}} \sigma_{k}(z),
$$

where $Z_{h}$ is the set of leaves that are consistent with the sequence $h$. The interpretation of $V^{G}$ is not as natural as in the case of normal-form games, as it is not possible for an agent to play one sequence to the exclusion of all others; its possible actions will be partially determined by the actions of other agents. In this case, $V_{h}^{G}(\sigma)$ can be regarded as the portion of its payoff that agent $n$ receives for playing sequence $h$, unscaled by agent $n$ 's own probability of playing that sequence. As with normal-form games, the vector of bonuses is added directly to $V^{G}$, so $V^{G \oplus \boldsymbol{b}}=V^{G}+\boldsymbol{b}$.

The retraction operator $R$ for realization plans is defined in the same way as for normalform strategies: it takes a general vector and projects it onto the nearest point in the valid region of realization plans. The constraints defining this space are linear, as discussed in Section 2.2.1. We can therefore express them as a constraint matrix $C$ with $C \sigma=0$ for all valid profiles $\sigma$. In addition, all probabilities must be greater than or equal to zero. To calculate $\boldsymbol{w}$, we must find a $\sigma$ minimizing $(w-\sigma)^{T}(w-\sigma)$, the (squared) Euclidean distance between $\boldsymbol{w}$ and $\sigma$, subject to $C \sigma=\mathbf{0}$ and $\sigma \geq \mathbf{0}$. This is a quadratic program (QP), which can be solved efficiently using standard methods. The Jacobian of the retraction is easily computable from the set of active constraints.

The equilibrium characterization for realization plans is now surprisingly similar to that of mixed strategies in normal-form games; GW show that, as before, equilibria are characterized by $\sigma=R\left(\sigma+V^{G}(\sigma)\right)$, where now $R$ is the retraction for sequence form and $V^{G}$ is the deviation function. The continuation equation $F$ takes exactly the same form as well.

\subsubsection{Computation}

The key property of the reduced sequence-form strategy representation is that the deviation function is a multi-linear function of the extensive-form parameters, as shown in Equation (7). The elements of the Jacobian $\nabla V^{G}$ thus also have the same general structure. In particular, the element corresponding to sequence $h$ for agent $n$ and sequence $h^{\prime}$ for agent $n^{\prime}$ is

$$
\begin{aligned}
\nabla V_{h, h^{\prime}}^{G}(\sigma) & =\frac{\partial}{\partial \sigma_{h^{\prime}}} \sum_{z \in Z_{h}} G_{n}(z) \prod_{k \in N \backslash\{n\}} \sigma_{k}(z) \\
& =\sum_{z \in Z_{h, h^{\prime}}} G_{n}(z) \prod_{k \in N \backslash\left\{n, n^{\prime}\right\}} \sigma_{k}(z)
\end{aligned}
$$

where $Z_{h, h^{\prime}}$ is the set of leaves that are consistent with the sequences $h$ (for agent $n$ ) and $h^{\prime}$ (for agent $n^{\prime}$ ). $Z_{h, h^{\prime}}$ is the empty set (and hence $\nabla V^{G}=0$ ) if $h$ and $h^{\prime}$ are incompatible. Equation (8) is precisely analogous to Equation (6) for normal-form games. We have a sum over outcomes of the utility of the outcome multiplied by the strategy probabilities for all 


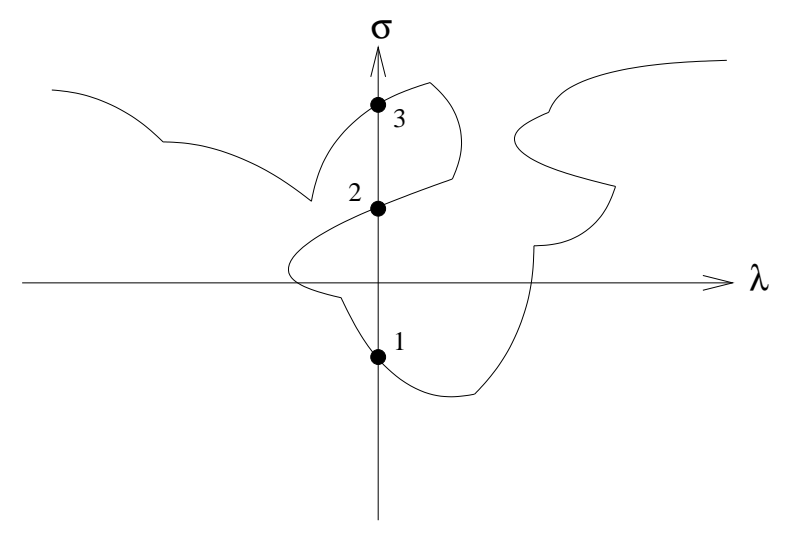

Figure 3: An abstract diagram of the path. The horizontal axis represents $\lambda$ and the vertical axis represents the space of strategy profiles (actually multidimensional). The algorithm starts on the right at $\lambda=1$ and follows the dynamical system until $\lambda=0$ at point 1 , where it has found an equilibrium of the original game. It can continue to trace the path and find the equilibria labeled 2 and 3.

other agents. Note that this sum is over the leaves of the tree, which may be exponentially numerous in the number of agents.

One additional subtlety, which must be addressed by any method for equilibrium computation in extensive-form games, relates to zero-probability actions. Such actions induce a probability of zero for entire trajectories in the tree, possibly leading to equilibria based on unrealizable threats. Additionally, for information sets that occur with zero probability, agents can behave arbitrarily without disturbing the equilibrium criterion, resulting in a continuum of equilibria and a possible bifurcation in the continuation path. This prevents our methods from converging. We therefore constrain all realization probabilities to be greater than or equal to $\epsilon$ for some small $\epsilon>0$. This is, in fact, a requirement for GW's equilibrium characterization to hold. The algorithm thus looks for an $\epsilon$-perfect equilibrium (Fudenberg \& Tirole, 1991): a strategy profile $\sigma$ in which each component is constrained by $\sigma_{s} \geq \epsilon$, and each agent's strategy is a best response among those satisfying the constraint. Note that this is entirely different from an $\epsilon$-equilibrium. An $\epsilon$-perfect equilibrium always exists, as long as $\epsilon$ is not so large as to make the set of legal strategies empty. An $\epsilon$-perfect equilibrium can be interpreted as an equilibrium in a perturbed game in which agents have a small probability of choosing an unintended action. A limit of $\epsilon$-perfect equilibria as $\epsilon$ approaches 0 is a perfect equilibrium (Fudenberg \& Tirole, 1991): a refinement of the basic notion of a Nash equilibrium. As $\epsilon$ approaches 0, the equilibria found by GW's algorithm therefore converge to an exact perfect equilibrium, by continuity of the variation in the continuation method path. Then for $\epsilon$ small enough, there is a perfect equilibrium in the vicinity of the found $\epsilon$-perfect equilibrium, which can easily be found with local search. 


\subsection{Path Properties}

In the case of normal-form games, GW show, using the structure theorem of Kohlberg and Mertens (1986), that the path of the algorithm is a one-manifold without boundary with probability one over all choices for $\boldsymbol{b}$. They provide an analogous structure theorem that guarantees the same property for extensive-form games. Figure 3(a) shows an abstract representation of the path followed by the continuation method. GW show that the path must cross the $\lambda=0$ hyperplane at least once, yielding an equilibrium. In fact, the path may cross multiple times, yielding many equilibria in a single run. As the path must eventually continue to the $\lambda=-\infty$ side, it will find an odd number of equilibria when run to completion.

In both normal-form and extensive-form games, the path is piece-wise polynomial, with each piece corresponding to a different support set of the strategy profile. These pieces are called support cells. The path is not smooth at cell boundaries due to discontinuities in the Jacobian of the retraction operator, and hence in $\nabla_{\boldsymbol{w}} F$, when the support changes. Care must be taken to step up to these boundaries exactly when following the path; at this point, the Jacobian for the new support can be calculated and the path can be traced into the new support cell.

In the case of two agents, the path is piece-wise linear and, rather than taking steps, the algorithm can jump from corner to corner along the path. When this algorithm is applied to a two-agent game and a particular bonus vector is used (in which only a single entry is nonzero), the steps from support cell to support cell that the algorithm takes are identical to the pivots of the Lemke-Howson algorithm (Lemke \& Howson, 1964) for two-agent generalsum games, and the two algorithms find precisely the same set of solutions (Govindan \& Wilson, 2002). Thus, the continuation method is a strict generalization of the LemkeHowson algorithm that allows different perturbation rays and games of more than two agents.

This process is described in more detail in the pseudo-code for the algorithm, presented in Figure 4.

\subsection{Computational Issues}

Guarantees of convergence apply only as long as we stay on the path defined by the dynamical system of the continuation method. However, for computational purposes, discrete steps must be taken. As a result, error inevitably accumulates as the path is traced, so that $F$ becomes slightly non-zero. GW use several simple techniques to combat this problem. We adopt their techniques, and introduce one of our own: we employ an adaptive step size, taking smaller steps when error accumulates quickly and larger ones when it does not. When $F$ is nearly linear (as it is, for example, when very few actions are in the support of the current strategy profile), this technique speeds computation significantly.

GW use two different techniques to remove error once it has accumulated. Suppose we are at a point $(w, \lambda)$ and we wish to minimize the magnitude of $F(w, \lambda)=w-V^{G}(R(w))+$ $\lambda \boldsymbol{b}+R(w)$. There are two values we might change: $w$, or $\lambda \boldsymbol{b}$. We can change the first without affecting the guarantee of convergence, so every few steps we run a local Newton method search for a $w$ minimizing $|F(w, \lambda)|$. If this search does not decrease error sufficiently, then we perform what GW call a "wobble": we change the perturbation vector ("wobble" the 
continuation path) to make the current solution consistent. If we set $\boldsymbol{b}=\left[w-V^{G}(R(w))-\right.$ $R(w)] / \lambda$, the equilibrium characterization equation is immediately satisfied. Changing the perturbation vector invalidates any theoretical guarantees of convergence. However, it is nonetheless an attractive option because it immediately reduces error to zero. Both the local Newton method and the "wobbles" are described in more detail by Govindan and Wilson (2003).

These techniques can potentially send the algorithm into a cycle, and in practice they occasionally do. However, they are necessary for keeping the algorithm on the path. If the algorithm cycles, random restarts and a decrease in step size can improve convergence. More sophisticated path-following algorithms might also be used, and in general could improve the success rate and execution time of the algorithm.

\subsection{Iterated Polymatrix Approximation}

Because perturbed games may themselves have a large number of equilibria, and the path may wind back and forth through any number of them, the continuation algorithm can take a while to trace its way back to a solution to the original game. We can speed up the algorithm using an initialization procedure based on the iterated polymatrix approximation (IPA) algorithm of GW. A polymatrix game is a normal-form game in which the payoffs to an agent $n$ are equal to the sum of the payoffs from a set of two-agent games, each involving $n$ and another agent. Because polymatrix games are a linear combination of two-agent normal-form games, they reduce to a linear complementarity problem and can be solved quickly using the Lemke-Howson algorithm (Lemke \& Howson, 1964).

For each agent $n \in N$ in a polymatrix game, the payoff array is a matrix $B^{n}$ indexed by the actions of agent $n$ and of each other agent; for actions $a \in A_{n}$ and $a^{\prime} \in A_{n^{\prime}}, B_{a, a^{\prime}}^{n}$ is the payoff $n$ receives for playing $a$ in its game with agent $n^{\prime}$, when $n^{\prime}$ plays $a^{\prime}$. Agent $n$ 's total payoff is the sum of the payoffs it receives from its games with each other agent, $\sum_{n^{\prime} \neq n} \sum_{a \in A_{n}, a^{\prime} \in A_{n^{\prime}}} \sigma_{a} \sigma_{a^{\prime}} B_{a, a^{\prime}}^{n}$. Given a normal-form game $G$ and a strategy profile $\sigma$, we can construct the polymatrix game $P_{\sigma}$ whose payoff function has the same Jacobian at $\sigma$ as $G$ 's by setting

$$
B_{a, a^{\prime}}^{n}=\nabla V_{a, a^{\prime}}^{G}(\sigma) .
$$

The game $P_{\sigma}$ is a linearization of $G$ around $\sigma$ : its Jacobian is the same everywhere. GW show that $\sigma$ is an equilibrium of $G$ if and only if it is an equilibrium of $P_{\sigma}$. This follows from the equation $V^{G}(\sigma)=\nabla V^{G}(\sigma) \cdot \sigma /(|N|-1)$, which holds for all $\sigma$. To see why it holds, consider the single element indexed by $a \in A_{n}$ :

$$
\begin{aligned}
\left(\nabla V^{G}(\sigma) \cdot \sigma\right)_{a} & =\sum_{n^{\prime} \in N \backslash\{n\}} \sum_{a^{\prime} \in A_{n^{\prime}}} \sigma_{a^{\prime}} \sum_{\boldsymbol{t} \in A_{-n,-n^{\prime}}} G_{n}\left(a, a^{\prime}, \boldsymbol{t}\right) \prod_{k \in N \backslash\left\{n, n^{\prime}\right\}} \sigma_{\boldsymbol{t}_{k}} \\
& =\sum_{n^{\prime} \in N \backslash\{n\}} \sum_{\boldsymbol{t} \in A_{-n}} G_{n}(a, \boldsymbol{t}) \prod_{k \in N \backslash\{n\}} \sigma_{\boldsymbol{t}_{k}} \\
& =(|N|-1) V^{G}(\sigma)_{a} .
\end{aligned}
$$

The equilibrium characterization equation can therefore be written

$$
\sigma=R\left(\sigma+\nabla V^{G}(\sigma) \cdot \sigma(|N|-1)\right) .
$$


$G$ and $P_{\sigma}$ have the same value of $\nabla V$ at $\sigma$, and thus the same equilibrium characterization function. Then $\sigma$ satisfies one if and only if it satisfies the other.

We define the mapping $p: \Sigma \rightarrow \Sigma$ such that $p(\sigma)$ is an equilibrium for $P_{\sigma}$ (specifically, the first equilibrium found by the Lemke-Howson algorithm). If $p(\sigma)=\sigma$, then $\sigma$ is an equilibrium of $G$. The IPA procedure of Govindan and Wilson (2004) aims to find such a fixed point. It begins with a randomly chosen strategy profile $\sigma$, and then calculates $p(\sigma)$ by running the Lemke-Howson algorithm; it adjusts $\sigma$ toward $p(\sigma)$ using an approximate derivative estimate of $p$ built up over the past two iterations. If $\sigma$ and $p(\sigma)$ are sufficiently close, it terminates with an approximate equilibrium.

IPA is not guaranteed to converge. However, in practice, it quickly moves "near" a good solution. It is possible at this point to calculate a perturbed game close to the original game (essentially, one that differs from it by the same amount that $G$ 's polymatrix approximation differs from $G$ ) for which the found approximate equilibrium is in fact an exact equilibrium. The continuation method can then be run from this starting point to find an exact equilibrium of the original game. The continuation method is not guaranteed to converge from this starting point. However, in practice we have always found it to converge, as long as IPA is configured to search for high quality equilibrium approximations. Although there are no theoretical results on the required quality, IPA can refine the starting point further if the continuation method fails. Our results show that the IPA quick-start substantially reduces the overall running time of our algorithm.

We can in fact use any other approximate algorithm as a quick-start for ours, also without any guarantees of convergence. Given an approximate equilibrium $\sigma$, the inverse image of $\sigma$ under $R$ is defined by a set of linear constraints. If we let $w:=V^{G}(\sigma)+\sigma$, then we can use standard QP methods to retract $w$ to the nearest point $w^{\prime}$ satisfying these constraints, and let $\boldsymbol{b}:=w^{\prime}-w$. Then $\sigma=R\left(w^{\prime}\right)=R\left(V^{G}(\sigma)+\sigma+\boldsymbol{b}\right)$, so we are on a continuation method path. Alternatively, we can choose $\boldsymbol{b}$ by "wobbling", in which case we set $\boldsymbol{b}:=\left[w-V^{G}(R(w))-R(w)\right] / \lambda$.

\section{Exploiting Structure}

Our algorithm's continuation method foundation is the same for each game representation, but the calculation of $\nabla V^{G}$ in Step 2(b)i of the pseudo-code in Figure 4 is different for each and consumes most of the time. Both in normal-form and (in the worst case) in extensiveform games, it requires exponential time in the number of agents. However, as we show in this section, when using a structured representation such as a graphical game or a MAID, we can effectively exploit the structure of the game to drastically reduce the computational time required.

\subsection{Graphical Games}

Since a graphical game is also a normal-form game, the definition of the deviation function $V^{G}$ in Equation (4) is the same: $V_{a}^{G}(\sigma)$ is the payoff to agent $n$ for deviating from $\sigma$ to play a deterministically. However, due to the structure of the graphical game, the choice of strategy for an agent outside the family of $n$ does not affect agent $n$ 's payoff. This observation allows us to compute this payoff locally. 
For an input game $G$ :

1. Set $\lambda=1$, choose initial $\boldsymbol{b}$ and $\sigma$ either by a quick-start procedure (e.g., IPA) or by randomizing. Set $\boldsymbol{w}=V^{G}(\sigma)+\lambda \boldsymbol{b}+\sigma$.

2. While $\lambda$ is greater than some (negative) threshold (i.e., there is still a good chance of picking up another equilibrium):

(a) Initialize for the current support cell: set the steps counter to the number of steps we will take in crossing the cell, depending on the current amount of error. If $F$ is linear or nearly linear (if, for example, the strategy profile is nearly pure, or there are only 2 agents), set steps $=1$ so we will cross the entire cell.

(b) While steps $\geq 1$ :

i. Compute $\nabla V^{G}(\sigma)$.

ii. Set $\nabla_{\boldsymbol{w}} F(\boldsymbol{w}, \lambda)=I-\left(\nabla V^{G}(\sigma)+I\right) \nabla R(\boldsymbol{w})$ (we already know $\nabla_{\lambda} F=-\boldsymbol{b}$ ). Set $d \boldsymbol{w}=$ $\operatorname{adj}\left(\nabla_{\boldsymbol{w}} F\right) \cdot \boldsymbol{b}$ and $d \lambda=\operatorname{det}\left(\nabla_{\boldsymbol{w}} F\right)$. These satisfy Equation (3).

iii. Set $\delta$ equal to the distance we'd have to go in the direction of $d \boldsymbol{w}$ to reach the next support boundary. We will scale $d \boldsymbol{w}$ and $d \lambda$ by $\delta /$ steps.

iv. If $\lambda$ will change signs in the course of the step, record an equilibrium at the point where it is 0 .

v. Set $\boldsymbol{w}:=\boldsymbol{w}+d \boldsymbol{w}(\delta /$ steps $)$ and $\lambda:=\lambda+d \lambda(\delta /$ steps $)$.

vi. If sufficient error has accumulated, use the local Newton method to find a $\boldsymbol{w}$ minimizing $|F(\boldsymbol{w}, \lambda)|$. If this does not reduce error enough, increase steps, thereby decreasing step size. If we have already increased steps, perform a "wobble" and reassign $\boldsymbol{b}$.

vii. Set steps $:=$ steps -1 .

Figure 4: Pseudo-code for the cont algorithm.

\subsubsection{The Jacobian for Graphical Games}

We begin with the definition of $V^{G}$ for normal-form games (modified slightly to account for the local payoff arrays). Recall that $A_{-n}^{f}$ is the set of action profiles of agents in Fam $_{n}$ other than $n$, and let $A_{-F_{a m}}$ be the set of action profiles of agents not in $F_{a m}$. Then we can divide a sum over full action profiles between these two sets, switching from the normal-form version of $G^{n}$ to the graphical game version of $G^{n}$, as follows:

$$
\begin{aligned}
V_{a}^{G}(\sigma) & =\sum_{\boldsymbol{t} \in A_{-n}} G_{n}(a, \boldsymbol{t}) \prod_{k \in N \backslash\{n\}} \sigma_{\boldsymbol{t}_{k}} \\
& =\sum_{\boldsymbol{u} \in A_{-n}^{f}} G_{n}(a, \boldsymbol{u}) \prod_{k \in F a m_{n} \backslash\{n\}} \sigma_{\boldsymbol{u}_{k}} \sum_{\boldsymbol{v} \in A_{-F a m_{n}}} \prod_{j \in N \backslash F a m_{n}} \sigma_{\boldsymbol{v}_{j}} .
\end{aligned}
$$

Note that the latter sum and product simply sum out a probability distribution, and hence are always equal to 1 due to the constraints on $\sigma$. They can thus be eliminated without changing the value $V^{G}$ takes on valid strategy profiles. However, their partial derivatives with respect to strategies of agents not in $\mathrm{Fam}_{n}$ are non-zero, so they enter into the computation of $\nabla V^{G}$.

Suppose we wish to compute a row in the Jacobian matrix corresponding to action $a$ of agent $n$. We must compute the entries for each action $a^{\prime}$ of each agent $n^{\prime} \in N$. In the trivial case where $n^{\prime}=n$ then $\nabla V_{a, a^{\prime}}^{G}=0$, since $\sigma_{a}$ does not appear anywhere in the expression for $V_{a}^{G}(\sigma)$. We next compute the entries for each action $a^{\prime}$ of each other agent $n^{\prime} \in$ Fam $_{n}$. 
In this case,

$$
\begin{aligned}
\nabla V_{a, a^{\prime}}^{G}(\sigma) & =\frac{\partial}{\partial \sigma_{a^{\prime}}} \sum_{\boldsymbol{u} \in A_{-n}^{f}} G_{n}(a, \boldsymbol{u}) \prod_{k \in F a m_{n} \backslash\{n\}} \sigma_{\boldsymbol{u}_{k}} \sum_{\boldsymbol{v} \in A_{-F a m_{n}}} \prod_{j \in N \backslash F a m_{n}} \sigma_{\boldsymbol{v}_{j}} \\
& =\sum_{\boldsymbol{u} \in A_{-n}^{f}} G_{n}(a, \boldsymbol{u}) \frac{\partial}{\partial \sigma_{a^{\prime}}} \prod_{k \in F a m_{n} \backslash\{n\}} \sigma_{\boldsymbol{u}_{k}} \cdot 1 \\
& =\sum_{\boldsymbol{t} \in A_{-n, n^{\prime}}^{f}} G_{n}\left(a, a^{\prime}, \boldsymbol{t}\right) \prod_{k \in F_{n} \backslash\left\{n, n^{\prime}\right\}} \sigma_{\boldsymbol{t}_{k}}, \quad \text { if } n^{\prime} \in \text { Fam }_{n} .
\end{aligned}
$$

We next compute the entry for a single action $a^{\prime}$ of an agent $n^{\prime} \notin F a m_{n}$. The derivative in Equation (11) takes a different form in this case; the variable in question is in the second summation, not the first, so that we have

$$
\begin{aligned}
\nabla V_{a, a^{\prime}}^{G}(\sigma) & =\frac{\partial}{\partial \sigma_{a^{\prime}}} \sum_{\boldsymbol{u} \in A_{-n}^{f}} G_{n}(a, \boldsymbol{u}) \prod_{k \in F a m_{n} \backslash\{n\}} \sigma_{\boldsymbol{u}_{k}} \sum_{\boldsymbol{v} \in A_{-F a m_{n}}} \prod_{j \in N \backslash F a m_{n}} \sigma_{\boldsymbol{v}_{j}} \\
& =\sum_{\boldsymbol{u} \in A_{-n}^{f}} G_{n}(a, \boldsymbol{u}) \prod_{k \in F a m_{n} \backslash\{n\}} \sigma_{\boldsymbol{u}_{k}} \sum_{\boldsymbol{v} \in A_{-F a m_{n}}} \frac{\partial}{\partial \sigma_{a^{\prime}}} \prod_{j \in N \backslash F a m_{n}} \sigma_{\boldsymbol{v}_{j}} \\
& =\sum_{\boldsymbol{u} \in A_{-n}^{f}} G_{n}(a, \boldsymbol{u}) \prod_{k \in F a m_{n} \backslash\{n\}} \sigma_{\boldsymbol{u}_{k}} \cdot 1, \quad \text { if } n^{\prime} \notin F a m_{n} .
\end{aligned}
$$

Notice that this calculation does not depend on $a^{\prime}$; therefore, it is the same for each action of each other agent not in $\mathrm{Fam}_{n}$. We need not compute any more elements of the row. We can copy this value into all other columns of actions belonging to agents not in $\mathrm{Fam}_{n}$.

\subsubsection{Computational Complexity}

Due to graphical game structure, the computation of $\nabla V^{G}(\sigma)$ takes time exponential only in the maximal family size of the game, and hence takes time polynomial in the number of agents if the family size is constant. In particular, our methods lead to the following theorem about the complexity of the continuation method for graphical games.

Theorem 10. The time complexity of computing the Jacobian of the deviation function $\nabla V^{G}(\sigma)$ for a graphical game is $O\left(f d^{f}|N|+d^{2}|N|^{2}\right)$, where $f$ is the maximal family size and $d$ is the maximal number of actions per agent.

Proof. Consider a single row in the Jacobian, corresponding to a single action $a$ owned by a single agent $n$. There are at most $d(f-1)$ entries in the row for actions owned by other members of $F_{a m}$. For one such action $a^{\prime}$, the computation of the Jacobian element $\nabla V_{a, a^{\prime}}^{G}$ according to Equation (12) takes time $O\left(d^{f-2}\right)$. The total cost for all such entries is therefore $O\left((f-1) d^{f-1}\right)$. There are then at most $d(|N|-f)$ entries for actions owned by non-familymembers. The value of $\nabla V_{a, a^{\prime}}^{G}$ for each such $a^{\prime}$ is the same. It can be calculated once in time $O\left(d^{f-1}\right)$, then copied across the row in time $d(|N|-f)$. All in all, the computational cost for the row is $O\left(f d^{f-1}+d|N|\right)$. There are at most $d|N|$ rows, so the total computational cost is $O\left(|N| f d^{f}+d^{2}|N|^{2}\right)$. 


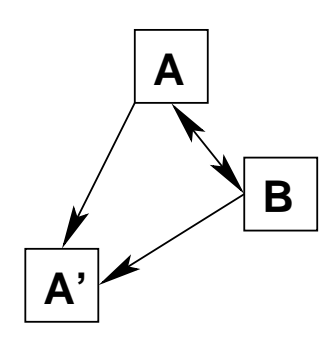

(a)

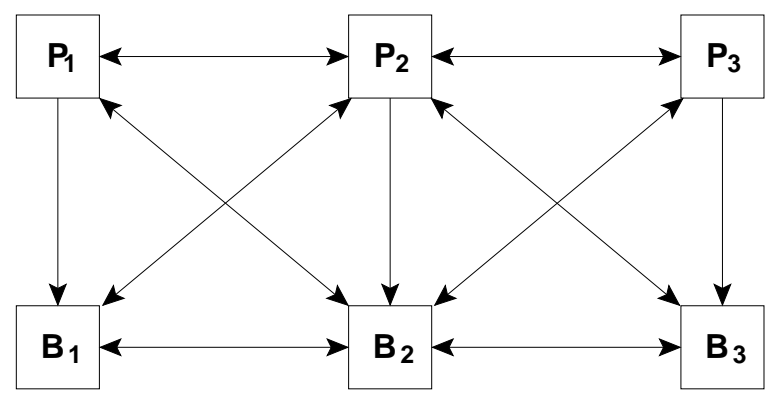

(b)

Figure 5: The strategic relevance graphs for the MAIDs in (a) Figure 2(a) and (b) Figure $2(b)$.

Each iteration of the algorithm calculates $\nabla V^{G}(\sigma)$ once; we have therefore proved that a single iteration takes time polynomial in $|N|$ if $f$ is constant (in fact, matrix operations make the complexity cubic in $|N|)$. However, as for normal-form games, there are no theoretical results about how many steps of the continuation method are required for convergence.

\subsection{MAIDs}

For graphical games, the exploitation of structure was straightforward. We now turn to the more difficult problem of exploiting structure in MAIDs. We take advantage of two distinct sets of structural properties. The first, a coarse-grained structural measure known as strategic relevance (Koller \& Milch, 2001), has been used in previous computational methods. After decomposing a MAID according to strategic relevance relations, we can exploit finer-grained structure by using the extensive-form continuation method of GW to solve each component's equivalent extensive-form game. In the next two sections, we describe these two kinds of structure.

\subsubsection{Strategic Relevance}

Intuitively, a decision node $D_{n}^{i}$ is strategically relevant to another decision node $D_{n^{\prime}}^{j}$ if agent $n^{\prime}$, in order to optimize its decision rule at $D_{n^{\prime}}^{j}$, needs to know agent $n$ 's decision rule at $D_{n}^{i}$. The relevance relation induces a directed graph known as the relevance graph, in which only decision nodes appear and an edge from node $D_{n^{\prime}}^{j}$ to node $D_{n}^{i}$ is present iff $D_{n}^{i}$ is strategically relevant to $D_{n^{\prime}}^{j}$. In the event that the relevance graph is acyclic, the decision rules can be optimized sequentially in any reverse topological order; when all the children of a node $D_{n}^{i}$ have had their decision rules set, the decision rule at $D_{n}^{i}$ can be optimized without regard for any other nodes.

When cycles exist in the relevance graph, however, further steps must be taken. Within a strongly connected component (SCC), a set of nodes for which a directed path between any two nodes exists in the relevance graph, decision rules cannot be optimized sequentiallyin any linear ordering of the nodes in the SCC, some node must be optimized before one 
of its children, which is impossible. Koller and Milch (2001) show that a MAID can be decomposed into SCCs, which can then be solved individually.

For example, the relevance graph for the MAID in Figure 2(a), shown in Figure 5(a), has one SCC consisting of $A$ and $B$, and another consisting of $A^{\prime}$. In this MAID, we would first optimize the decision rule at $A^{\prime}$, as the optimal decision rule at $A^{\prime}$ does not rely on the decision rules at $A$ and $B$ - when she makes her decision at $A^{\prime}$, Alice already knows the actions taken at $A$ and $B$, so she does not need to know the decision rules that led to them. Then we would turn $A^{\prime}$ into a chance node with CPD specified by the optimized decision rule and optimize the decision rules at $A^{\prime}$ and $B$. The relevance graph for Figure 2(b), shown in Figure 5(b), forms a single strongly connected component.

The computational method of Koller and Milch (2001) stops at strategic relevance: each SCC is converted into an equivalent extensive-form game and solved using standard methods. Our algorithm can be viewed as an augmentation of their method: after a MAID has been decomposed into SCCs, we can solve each of these SCCs using our methods, taking advantage of finer-grained MAID structure within them to find equilibria more efficiently. The MAIDs on which we test our algorithms (including the road MAID in Figure 2b) all have strongly connected relevance graphs, so they cannot be decomposed (see Figure 5b and Figure 10).

\subsubsection{ThE JACOBIAN FOR MAIDs}

A MAID is equivalent to an extensive-form game, so its deviation function $V^{G}$ is the same one defined in Equation (8). Now, however, we can compute the payoffs that make up the Jacobian $\nabla V^{G}$ more efficiently. Consider a payoff $G_{n}(z)$ to agent $n$ for outcome $z$. The outcome $z$ is simply an assignment $\boldsymbol{x}$ to all of the variables in the MAID. The realization probability $\sigma_{n}(z)$ is the product of the probabilities for the decisions of agent $n$ in the assignment $\boldsymbol{x}$, so the product $\prod_{k \in N} \sigma_{k}(z)$ of all realization probabilities is simply the joint probability of the assignment. The expected payoff agent $n$ will receive under the strategy profile $\sigma, \sum_{z \in Z} G_{n}(z) \prod_{k \in N} \sigma_{k}(z)$, is therefore an expectation of $G_{n}(z)$. The expectation is with respect to the distribution $P_{\sigma}$ defined by the Bayesian network $\mathcal{B}_{\sigma}$ whose structure is the same as the MAID, with decision node CPDs determined by $\sigma$.

The entries of $\nabla V^{G}$ are not strictly expected payoffs, however. Equation (8) can be rewritten as

$$
\nabla V_{h, h^{\prime}}^{G}(\sigma)=\sum_{z \in Z_{h, h^{\prime}}} \frac{G_{n}(z) \prod_{k \in N} \sigma_{k}(z)}{\sigma_{n}(z) \sigma_{n^{\prime}}(z)} .
$$

The expectation is of the quantity $G_{n}(z) /\left[\sigma_{n}(z) \sigma_{n^{\prime}}(z)\right]$. The payoff $G_{n}(z)$ is the sum of agent $n$ 's utility nodes. Due to linearity of expectation, we can perform the computation separately for each of agent $n$ 's utility nodes, and then simply add up the separate contributions.

We can therefore restrict our attention to computing the contribution of a single utility node $U_{n}$ for each agent $n$. Furthermore, the value of $\sigma_{n}(z)$ depends only on the values of the set of nodes $\boldsymbol{D}_{n}$ consisting of $n$ 's decision nodes and their parents. Thus, instead of computing the probabilities for all assignments to all variables, we need only compute the marginal joint distribution over $U_{n}, \boldsymbol{D}_{n}$, and $\boldsymbol{D}_{n^{\prime}}$. From this distribution, we can compute the contribution of $U_{n}$ to the expectation in Equation (14) for every pair of terminal sequences belonging to agents $n$ and $n^{\prime}$. 


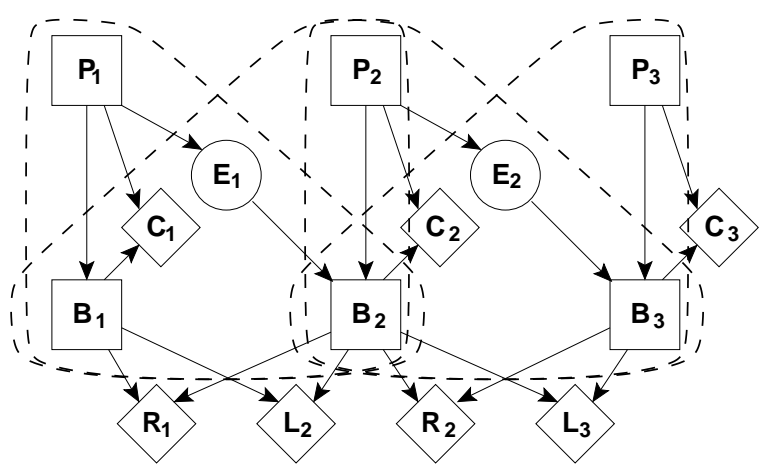

(a)

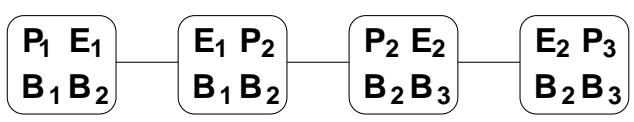

(b)

Figure 6: (a) A two-stage road MAID with three agents is shown divided into cliques. Each of the four cliques is surrounded by a dashed line, and has three decision nodes and a chance node. (b) The resultant clique tree.

\subsubsection{Using Bayesian Network Inference}

Our analysis above reduces the required computations significantly. Rather than computing a separate expectation for every pair of sequences $h, h^{\prime}$, as might at first have seemed necessary, we need only compute one marginal joint distribution over the variables in $\left\{U_{n}\right\} \cup$ $\boldsymbol{D}_{n} \cup \boldsymbol{D}_{n^{\prime}}$ for every pair of agents $n, n^{\prime}$. This marginal joint distribution is the one defined by the Bayesian network $\mathcal{B}_{\sigma}$. Naively, this computation requires that we execute Bayesian network inference $|N|^{2}$ times: once for each ordered pair of agents $n, n^{\prime}$. In fact, we can exploit the structure of the MAID to perform this computation much more efficiently. The basis for our method is the standard clique tree algorithm of Lauritzen and Spiegelhalter (1998). The clique tree algorithm is fairly complex, and a detailed presentation is outside the scope of this paper. We choose to treat the algorithm as a black box, describing only those of its properties that are relevant to understanding how it is used within our computation. We note that these details suffice to allow our method to be implemented using one of the many off-the-shelf implementations of the clique tree algorithm. A reader wishing to understand the clique tree algorithm or its derivation in more detail is referred to the reference by Cowell et al. (1999) for a complete description.

A clique tree for a Bayesian network $\mathcal{B}$ is a data structure defined over an undirected tree with a set of nodes $\mathcal{C}$. Each node $C_{i} \in \mathcal{C}$ corresponds to some subset of the variables in $\mathcal{B}$, typically called a clique. The clique tree satisfies certain important properties. It must be family preserving: for each node $X$ in $\mathcal{B}$, there exists a clique $C_{i} \in \mathcal{C}$ such that $\left(X \cup P a_{X}\right) \subseteq C_{i}$. It also satisfies a separation requirement: if $C_{2}$ lies on the unique path from $C_{1}$ to $C_{3}$, then, in the joint distribution defined by $\mathcal{B}$, the variables in $C_{1}$ must be conditionally independent of those in $C_{3}$ given those in $C_{2}$.

The division of the 3-agent road MAID into cliques is shown in Figure 7.2.3(a). This MAID has 4 cliques. Notice that every family is contained in a clique (including the families of chance nodes and utility nodes). The clique tree for this MAID is shown in Figure 7.2.3(b). 
Each clique maintains a data structure called a potential, a table with an entry for each joint assignment to the variables in the clique. A table of this sort is more generally called a factor. Inference algorithms typically use two basic operations on factors: factor product, and factor marginalization. If $\mathcal{F}$ and $\mathcal{G}$ are two factors over the (possibly overlapping) sets of variables $\boldsymbol{X}$ and $\boldsymbol{Y}$, respectively, then we can define the product $\mathcal{F} \mathcal{G}$ to be a new factor over $\boldsymbol{X} \cup \boldsymbol{Y}$. The entry in $\mathcal{F G}$ for a particular assignment to the variables in $\boldsymbol{X} \cup \boldsymbol{Y}$ is the product of the entries in $\mathcal{F}$ and $\mathcal{G}$ corresponding to the restriction of the assignment to $\boldsymbol{X}$ and $\boldsymbol{Y}$, respectively. This notion of multiplication corresponds to the way that conditional probability distributions are multiplied. We can also marginalize, or sum, a variable $X$ out of a factor $\mathcal{F}$ over $\boldsymbol{X}$ in the same way in which we would sum a variable out of a joint probability distribution. The result is a factor $\sum_{X} \mathcal{F}$ over the variables in $\boldsymbol{X} \backslash\{X\}$. The entry for a particular assignment to the variables in $\sum_{X} \mathcal{F}$ is equal to the sum of all entries in $\mathcal{F}$ compatible with that assignment — one for each value of $X$.

Because a factor has an entry for every joint assignment to its variables, the size of the potential for $C_{i}$ is exponential in $\left|C_{i}\right|$. The clique tree inference algorithm proceeds by passing messages, themselves factors, from one clique to another in the tree. The messages are used to update the potential in the receiving clique by factor multiplication. After a process in which messages have been sent in both directions over each edge in the tree, the tree is said to be calibrated; at this point, the potential of every clique $C_{i}$ contains precisely the joint distribution over the variables in $C_{i}$ according to $\mathcal{B}$ (for details, we refer to the reference by Cowell et al., 1999).

We can use the clique tree algorithm to perform inference over $\mathcal{B}_{\sigma}$. Consider the final decision node for agent $n$. Due to the perfect recall assumption, all of $n$ 's previous decisions and all of their parents are also parents of this decision node. The family preservation property therefore implies that $\boldsymbol{D}_{n}$ is fully contained in some clique. It also implies that the family of each utility node is contained in a clique. The expectation of Equation (14) thus requires the computation of the joint distribution over three cliques in the tree: the one containing $P a_{U_{n}}$, the one containing $\boldsymbol{D}_{n}$, and the one containing $\boldsymbol{D}_{n^{\prime}}$. We need to compute this joint distribution for every pair of agents $n, n^{\prime}$.

The first key insight is that we can reduce this problem to one of computing the joint marginal distribution for all pairs of cliques in the tree. Assume we have computed $P_{\mathcal{B}}\left(C_{i}, C_{j}\right)$ for every pair of cliques $C_{i}, C_{j}$. Now, consider any triple of cliques $C_{i}, C_{j}, C_{k}$. There are two cases: either one of these cliques is on the path between the other two, or not. In the first case, assume without loss of generality that $C_{j}$ is on the path from $C_{i}$ to $C_{k}$. In this case, by the separation requirement, we have that $P_{\mathcal{B}}\left(C_{i}, C_{j}, C_{k}\right)=P_{\mathcal{B}}\left(C_{i}, C_{j}\right) P_{\mathcal{B}}\left(C_{j}, C_{k}\right) / P_{\mathcal{B}}\left(C_{j}\right)$. In the second case, there exists a unique clique $C^{*}$ that lies on the path between any pair of these cliques. Again, by the separation property, $C^{*}$ renders these cliques conditionally independent, so we can compute

$$
P_{\mathcal{B}}\left(C_{i}, C_{j}, C_{k}\right)=\sum_{C^{*}} \frac{P_{\mathcal{B}}\left(C_{i}, C^{*}\right) P_{\mathcal{B}}\left(C_{j}, C^{*}\right) P_{\mathcal{B}}\left(C_{k}, C^{*}\right)}{P_{\mathcal{B}}\left(C^{*}\right)^{2}} .
$$

Thus, we have reduced the problem to one of computing the marginals over all pairs of cliques in a calibrated clique-tree. We can use dynamic programming to execute this process efficiently. We construct a table that contains $P_{\mathcal{B}}\left(C_{i}, C_{j}\right)$ for each pair of cliques $C_{i}, C_{j}$. We construct the table in order of length of the path from $C_{i}$ to $C_{j}$. The base case is when $C_{i}$ and 
$C_{j}$ are adjacent in the tree. In this case, we have that $P_{\mathcal{B}}\left(C_{i}, C_{j}\right)=P_{\mathcal{B}}\left(C_{i}\right) P_{\mathcal{B}}\left(C_{j}\right) / P_{\mathcal{B}}\left(C_{i} \cap\right.$ $C_{j}$ ). The probability expressions in the numerator are simply the clique potentials in the calibrated tree. The denominator can be obtained by marginalizing either of the two cliques. In fact, this expression is computed as a byproduct of the calibration process, so the marginalization is not required. For cliques $C_{i}$ and $C_{j}$ that are not adjacent, we let $C_{k}$ be the node adjacent to $C_{j}$ on the path from $C_{i}$ to $C_{j}$. The clique $C_{k}$ is one step closer to $C_{i}$, so, by construction, we have already computed $P\left(C_{i}, C_{k}\right)$. We can now apply the separation property again:

$$
P_{\mathcal{B}}\left(C_{i}, C_{j}\right)=\sum_{C_{k}} \frac{P_{\mathcal{B}}\left(C_{i}, C_{k}\right) P_{\mathcal{B}}\left(C_{k}, C_{j}\right)}{P_{\mathcal{B}}\left(C_{k}\right)}
$$

\subsubsection{Computational Complexity}

Theorem 11. The computation of $\nabla V^{G}(\sigma)$ can be performed in time $O\left(\ell^{2} d^{3}+u|N| d^{4}\right)$, where $\ell$ is the number of cliques in the clique tree for $G, d$ is the size of the largest clique (the number of entries in its potential), $|N|$ is the number of agents, and $u$ is the total number of utility nodes in the game.

Proof. The cost of calibrating the clique tree for $\mathcal{B}_{\sigma}$ is $O(\ell d)$. The cost of computing Equation (16) for a single pair of cliques is $O\left(d^{3}\right)$, as we must compute a factor over the variables in three cliques before summing out. We must perform this computation $O\left(\ell^{2}\right)$ times, once for each pair of cliques, for a total cost of $O\left(\ell^{2} d^{3}\right)$. We now compute marginal joint probabilities over triples of cliques $P a_{U_{n}^{i}}, \boldsymbol{D}_{n}, \boldsymbol{D}_{n^{\prime}}$ for every utility node $U_{n}^{i}$ and every agent $n^{\prime}$ other than $n$. There are $u(|N|-1)$ such triples. Computing a factor over the variables in three cliques may first require computing a factor over the variables in four cliques, at a cost of $O\left(d^{4}\right)$. Given this factor, computing the expected value of the utility node takes time $O\left(d^{3}\right)$, which does not affect the asymptotic running time. The total cost for computing all the marginal joint probabilities and expected utilities is therefore $O\left(u|N| d^{4}\right)$, and the total cost for computing $\nabla V^{G}(\sigma)$ is $O\left(\ell^{2} d^{3}+u|N| d^{4}\right)$.

With this method, we have shown that a single iteration in the continuation method can be accomplished in time exponential in the induced width of the graph - the number of variables in the largest clique in the clique tree. The induced width of the optimal clique tree - the one with the smallest maximal clique - is called the treewidth of the network. Although finding the optimal clique tree is, itself, an NP-hard problem, good heuristic algorithms are known (Cowell et al., 1999). In games where interactions between the agents are highly structured (the road MAID, for example), the size of the largest clique can be a constant even as the number of agents grows. In this case, the complexity of computing the Jacobian grows only quadratically in the number of cliques, and hence also in the number of agents. Note that the matrix adjoint operation takes time cubic in $m$, which is at least $|N|$, so a single step along the path actually has cubic computational cost. 


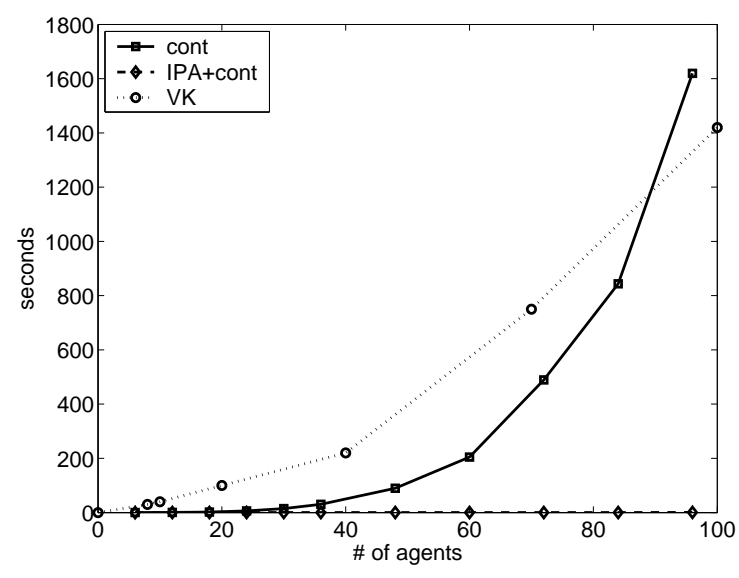

(a)

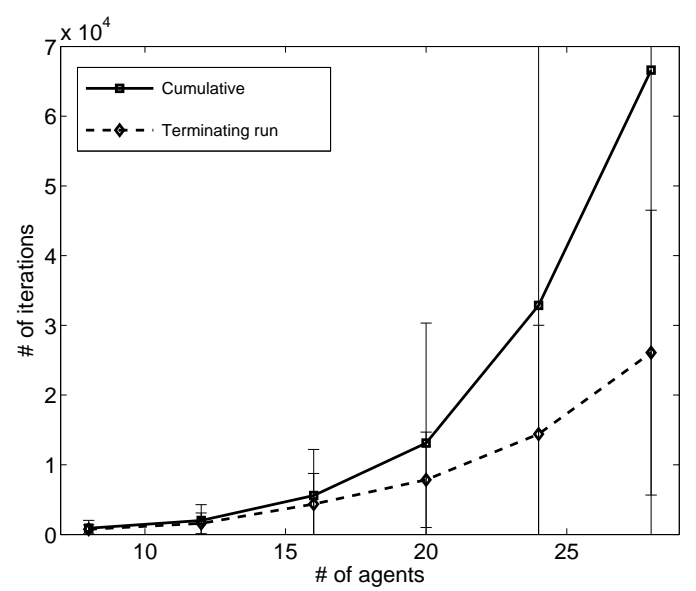

(c)

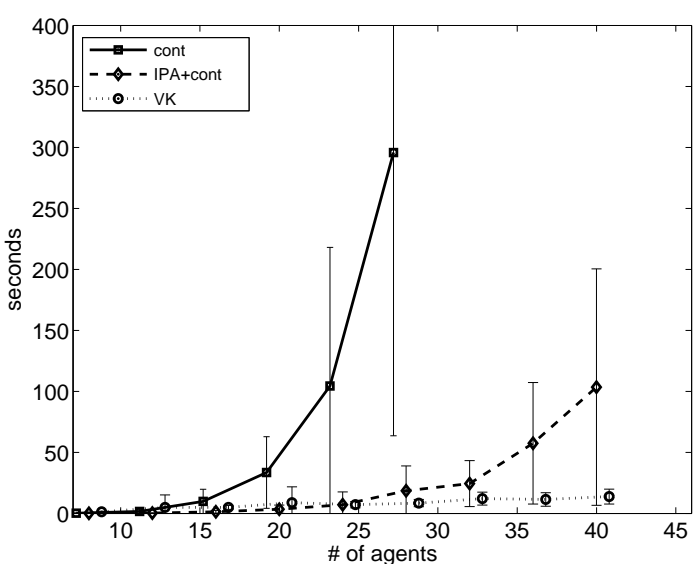

(b)

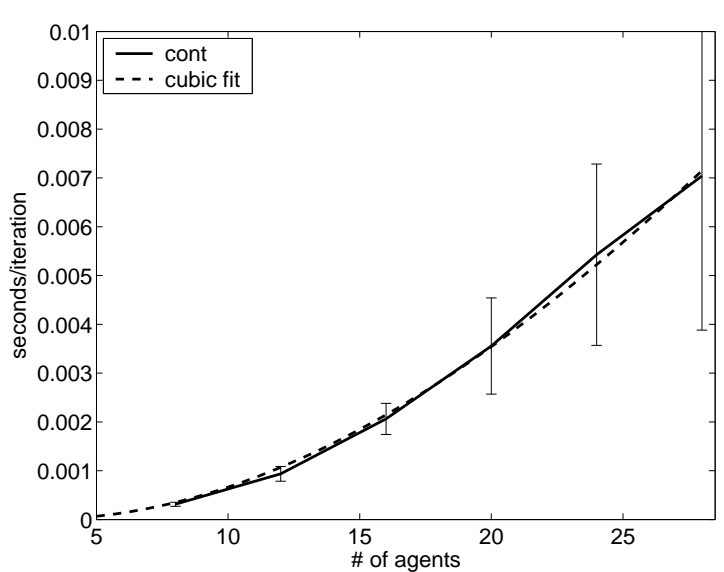

(d)

Figure 7: Results for 2-by- $L$ road game with rock-paper-scissors payoffs: (a) running time. Results for road game with random payoffs: (b) running time; (c) number of iterations of cont; (d) average time per iteration of cont.

\section{Results}

We performed run-time tests of our algorithms on a wide variety of both graphical games and MAIDs. Tests were performed on an Intel Xeon processor running at $3 \mathrm{GHz}$ with 2 GB of RAM, although the memory was never taxed during our calculations.

\subsection{Graphical Games}

For graphical games, we compared two versions of our algorithm: cont, the simple continuation method, and IPA+cont, the continuation method with IPA initialization. We tested the hybrid equilibrium refinement algorithm of Vickrey and Koller (2002) (VK hereafter) 


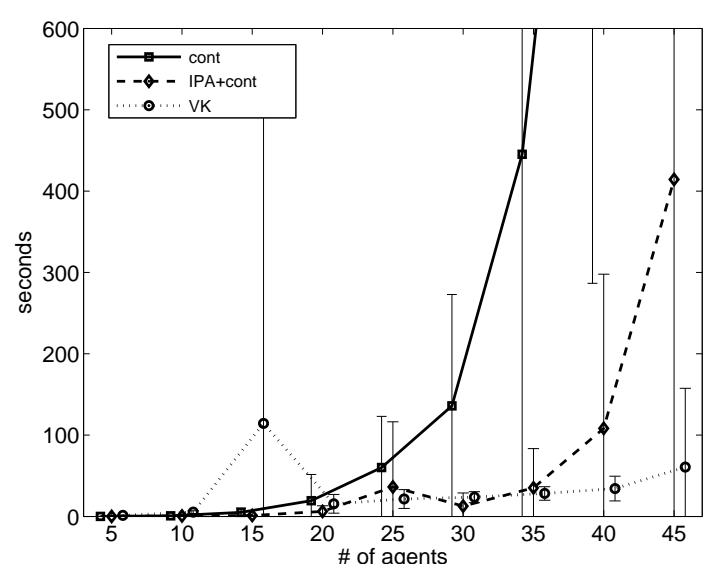

(a)

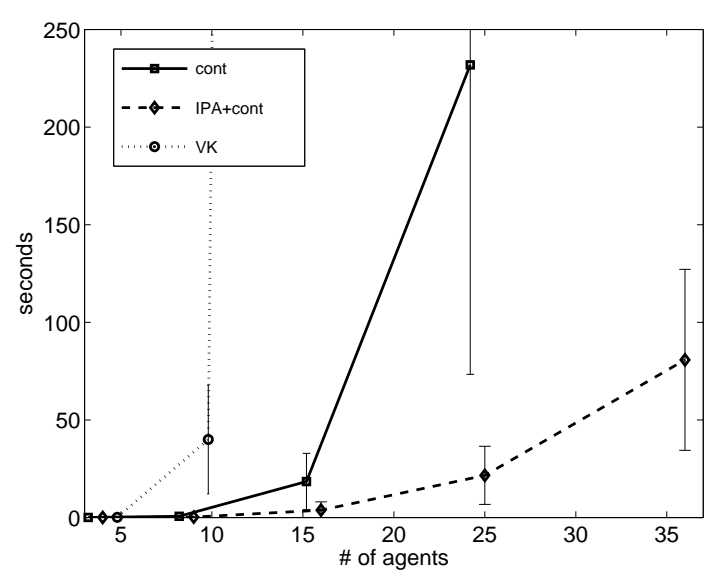

(c)

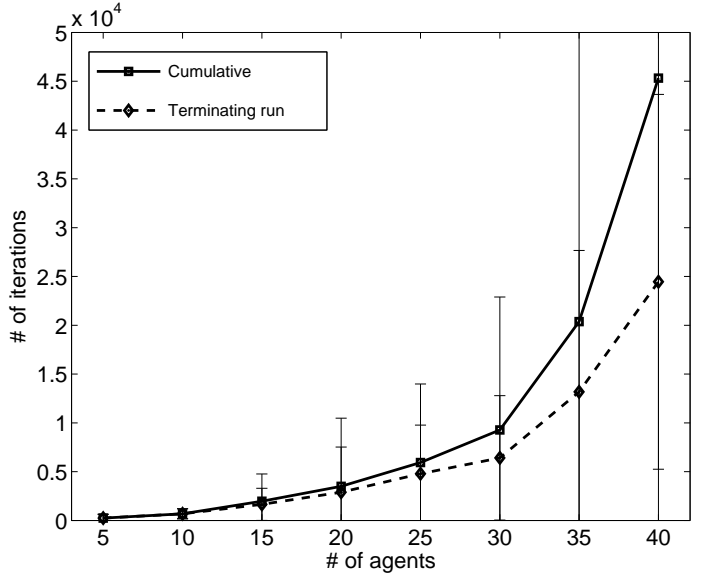

(b)

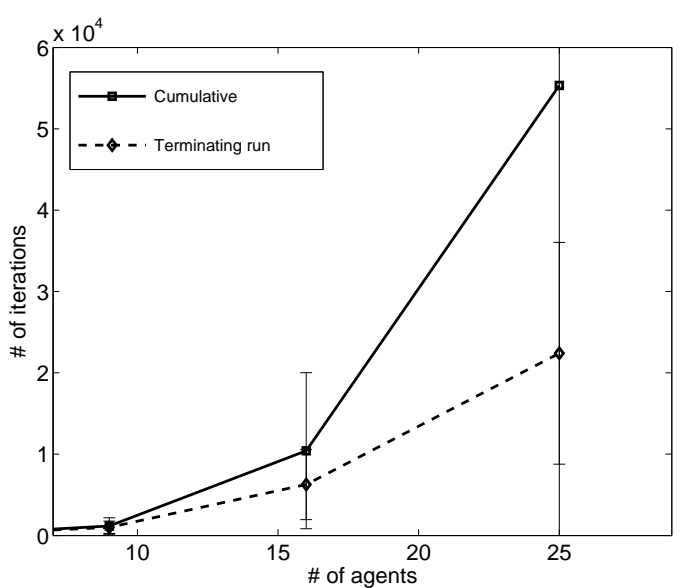

(d)

Figure 8: Results for ring game with random payoffs: (a) running time; (b) number of iterations of cont. Results for $L$-by- $L$ grid game with random payoffs: (c) running time; (d) number of iterations of cont.

for comparison, with the same parameters that they used. The VK algorithm only returns $\epsilon$-equilibria; no exact methods exist which are comparable to our own.

Our algorithms were run on two classes of games defined by Vickrey and Koller (2002) and two additional classes. The road game of Example 3, denoting a situation in which agents must build land plots along a road, is played on a 2-by- $L$ grid; each agent has three actions, and its payoffs depend only on the actions of its (grid) neighbors. Following VK, we ran our algorithm on road games with additive rock-paper-scissors payoffs: each agent's payoffs are a sum of payoffs from independent rock-paper-scissors games with each of its neighbors. This game is, in fact, a polymatrix game, and hence is very easy to solve using our methods. In order to test our algorithms on more typical examples, we experimented with 
road games in which the entries of the payoff matrix for each agent were chosen uniformly at random from $[0,1]$. We also experimented with a ring graph with three actions per agent and random payoffs. Finally, in order to test games with increasing treewidth, we experimented with grid games with random payoffs. These are defined in the same manner as the road games, except that the game graph is an $L$-by- $L$ grid.

For each class of games, we chose a set of game sizes to run on. For each, we selected (randomly in cases where the payoffs were random) a set of 20 test games to solve. We then solved each game using cont, IPA+cont, and VK. For cont, we started with a different random perturbation vector each time and recorded the time and number of iterations necessary to reach the first equilibrium. For IPA+cont, we started with a different initial strategy profile for IPA each time and recorded the total time for both IPA and cont to reach the first equilibrium.

All equilibria found by our algorithm had error at most $10^{-12}$, essentially machine precision. The hybrid refinement algorithm of VK found $\epsilon$-equilibria with average error of about $10^{-4}$ for road games with rock-paper-scissors payoffs, 0.01 for road games and grid games with random payoffs, and 0.03 for ring games with random payoffs, although the equilibria had error as high as 0.05 for road games and 0.1 for ring games.

For smaller games, the algorithms always converged to an equilibrium. In some larger games, cont or IPA detected that they had entered a cycle and terminated without finding an equilibrium. By maintaining a hash table of support cells they have passed through already, both cont and IPA are able to detect when they have entered a support cell for the second time. Although this is not a sure sign that they have entered a cycle, it is a strong indicator. When potential cycles were detected, the algorithms were restarted with new random initialization values. Note that cycles in the execution of cont can never arise if the algorithm does not stray from the path dictated by the theory of GW, so that random restarts reflect a failure to follow the path accurately.

When an equilibrium was eventually found, the cumulative time for all the random restarts was recorded. The error bars in the running time graphs show the variance due to the number of random restarts required, the choices of initialization values, and, for random games, the choice of game.

Random restarts were required in $29 \%$ of the games we tested. On average, 2.2 restarts were necessary for these games. Note that this figure is skewed by the larger games, which occasionally required many restarts; the largest games sometimes required 8 or 9 restarts. In a few large graphical games (10 random road games and 8 random ring games), IPA did not converge after 10 restarts; in these cases we did not record results for IPA+cont. cont always found an equilibrium within 10 restarts. Our results are shown in Figures $7(\mathrm{a}, \mathrm{b}, \mathrm{c}, \mathrm{d})$ and Figures 8(a,b,c).

For random roads, we also plotted the number of iterations and time per iteration for cont in Figures $7(\mathrm{c}, \mathrm{d})$. The number of iterations varies based both on the game and perturbation vector chosen. However, the time per iteration is almost exactly cubic, as predicted. We note that, when IPA was used as a quick-start, cont invariably converged immediately (within a second) - all of the time was spent in the IPA algorithm.

In the road games, our methods are more efficient for smaller games, but then become more costly. Due to the polymatrix nature of the rock-paper-scissors road games, the IPA+cont algorithm solves them immediately with the Lemke-Howson algorithm, and 
is therefore significantly less expensive than VK. In the random ring games, our algorithms are more efficient than VK for smaller games (up to 20-30 agents), with IPA+cont performing considerably better than cont. However, as with road games, the running time of our algorithms grows more rapidly than that of VK, so that for larger games, they become impractical. Nevertheless, our algorithms performed well in games with up to 45 agents and 3 actions per agent, which were previously intractable for exact algorithms. For the $L$-by- $L$ grid games, our algorithm performed much better than the VK algorithm (see Figures $8(\mathrm{c}, \mathrm{d}))$, with and without IPA quick-start. This reflects the fact that the running-time complexity of our algorithms does not depend on the treewidth of the graph.

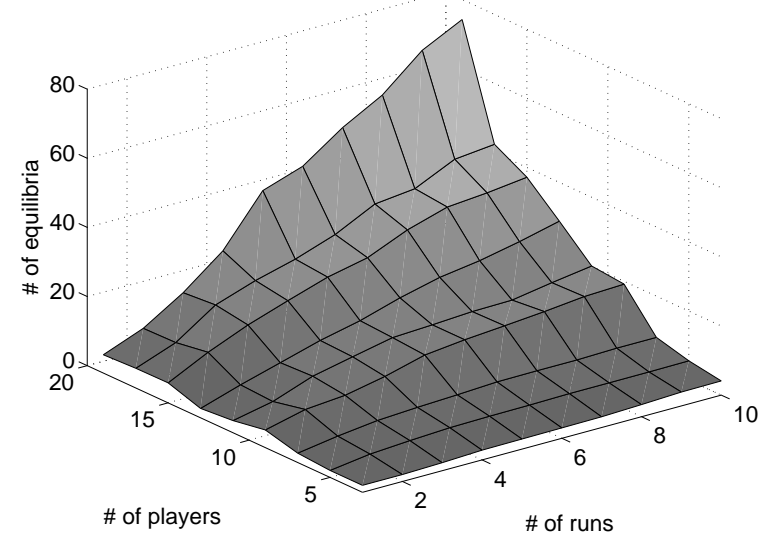

Figure 9: The number of unique equilibria found as a function of the size of the game and the number of runs of the algorithm, averaged over ten random ring games.

We also examined the number of equilibria found by the IPA+cont algorithm. We ran IPA+cont on the ring graphical game for differing numbers of agents. For each number of agents, we fixed 10 random games, ran the algorithm 10 times on each game, and recorded the cumulative number of unique equilibria found. The average number of equilibria found over the 10 games for each number of agents is plotted in figure 9. For small games (with presumably a small number of equilibria), the number of equilibria found quickly saturated. For large games, there was an almost linear increase in the number of equilibria found by each subsequent random restart, implying that each run of the algorithm produced a new set of solutions.

\subsection{MAIDs}

The previous computational method for MAIDs (Koller \& Milch, 2001) stopped at strategic relevance: each SCC was converted into an equivalent extensive-form game and solved using standard methods. Our algorithm takes advantage of further structure once a game has already been decomposed according to strategic relevance. All of our test cases were therefore selected to have relevance graphs consisting of a single strongly connected component. 


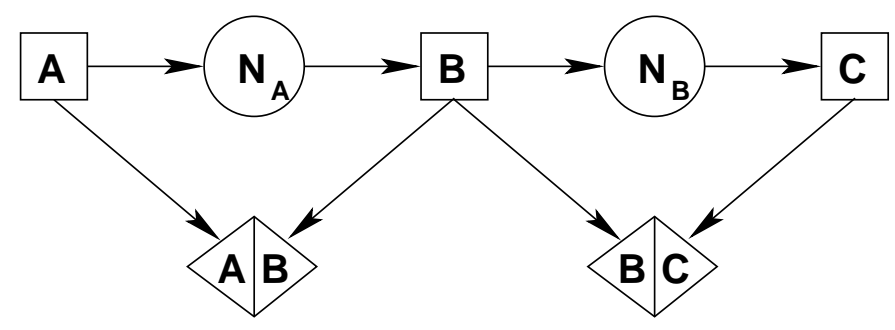

(a)

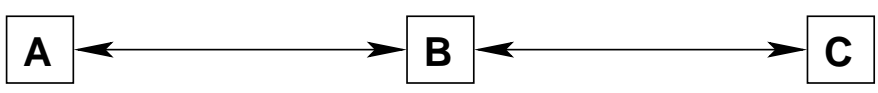

(b)

Figure 10: (a) The chain game and (b) its strategic relevance graph for the case of three agents (A, B, and $\mathrm{C})$.

In order to ascertain how much difference our enhancements made, we compared the results for our MAID algorithm, MAID cont, to those achieved by converting the game to extensive-form and running both EF cont, the extensive-form version of cont as specified by GW, and Gambit (McKelvey, McLennan, \& Turocy, 2004), a standard game theory software package. The time required for conversion to extensive form is not included in our results.

We ran our algorithms on two classes of games, with varying sizes. The first, to which we refer as the chain game, alternates between decision and chance nodes (see Figure 10). Each decision node belongs to a different agent. Each agent has two utility nodes, each connected to its own decision node and to a neighbor's (except for the end agents, who have one utility node for their single neighbor). There are three actions at each decision node. All probability tables and payoff matrices are chosen at uniformly at random. The second class is the two-stage road building game from Example 5, shown in Figure 2(b). In this class, we chose payoffs carefully, by hand, to ensure non-trivial mixed strategy equilibria.

We ran on chain games of all sizes between 2 and 21, and road games of all sizes between 2 and 9. For each size, we randomly selected 20 perturbation vectors and 20 games (all 20 road games were the same, since payoffs were set by hand, and all 20 chain games had payoffs randomly assigned). We then tested the algorithms on these games, initialized with these perturbation vectors, and averaged across test cases. The timing results appear in Figures 11(a,b). The error bars reflect variance due to the choice of game (in the chain games), the choice of perturbation vector, and the number of random restarts required.

In some cases, as with the graphical game tests, MAID cont failed to find an equilibrium, terminating early because it detected that it had entered a cycle. In these cases, it was restarted with a new perturbation vector until it successfully terminated. When an equilibrium was eventually found, the cumulative time for all the random restarts was recorded. Over the course of our test runs, only two chain games required a random restart. Both were of size 7. Our algorithms failed more frequently on road games; the spike for road games of size 8 reflects the fact that the games of this size required, on average, 1.2 


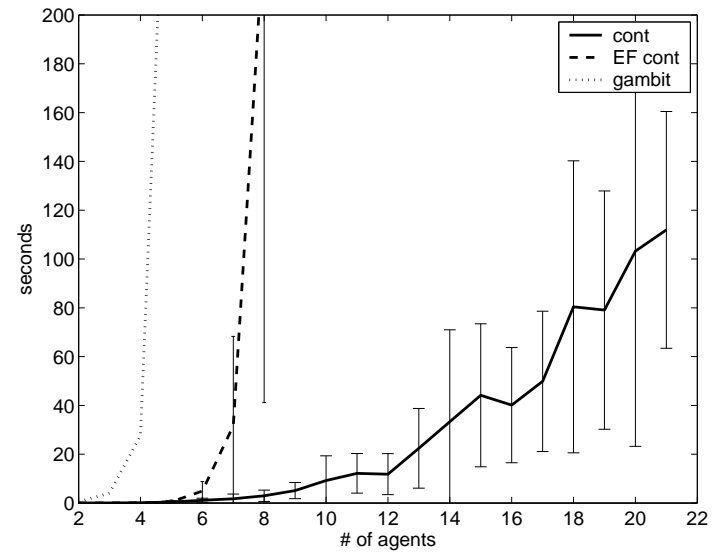

(a)

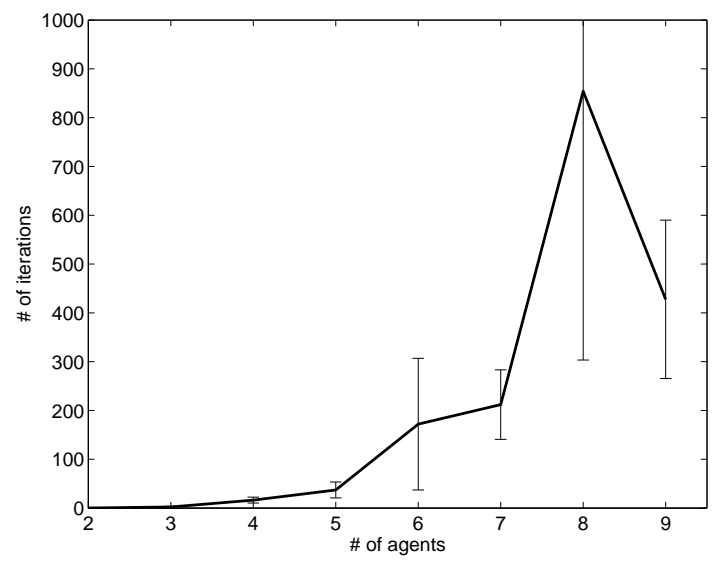

(c)

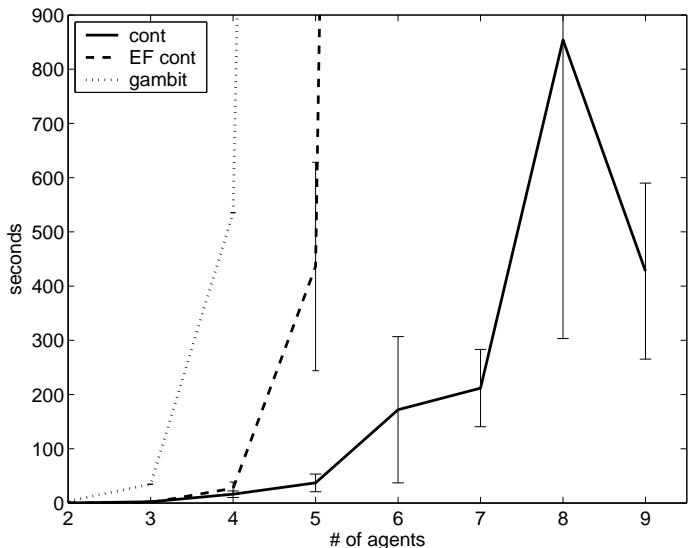

(b)

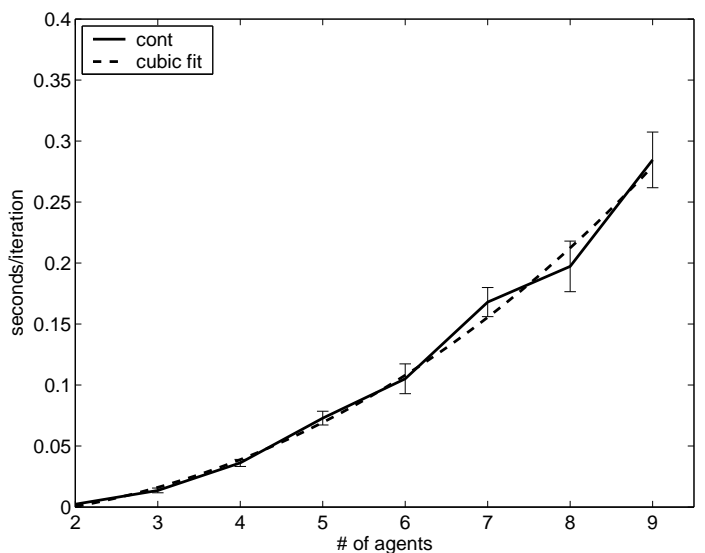

(d)

Figure 11: Results for MAIDs: (a) Running times for the chain MAID. Results for two-stage road MAID: (b) running time; (c) number of iterations; (d) time per iteration.

random restarts before an equilibrium was found. Strangely, MAID cont was much more successful on the road game of size 9 , succeeding without random restarts in all but two cases.

We tested Gambit and EF cont only on smaller games, because the time and memory requirements for testing on larger ones were beyond our means. Our results show that, while EF cont is a faster algorithm than Gambit for extensive-form games, it is inadequate for the larger MAIDs that we were able to solve with MAID cont. This is not at all surprising; a road game of size 9 has 26 decision or chance nodes, so the equivalent extensive-form game tree has $2^{26} \approx 67$ million outcome nodes. For MAIDs of this size, the Bayesian network inference techniques that we have used become necessary. 
For all MAIDs, realization probabilities were constrained to be at least $10^{-4}$ (i.e., we found $\epsilon$-perfect equilibria with $\epsilon=10^{-4}$ ). The accuracy of these equilibria was within $10^{-12}$, or machine precision.

As with graphical games, we recorded the number of iterations until convergence as well as the time per iteration for MAID cont. The results appear in Figures 11(c,d). The time per iteration is fit well by a cubic curve, in accordance with our theoretical predictions. The variance is primarily due to the execution of the retraction operator, whose running time depends on the number of strategies in the support.

\section{Discussion and Conclusions}

We have described here two adaptations of the continuation method algorithms of GW, for the purpose of accelerated execution on structured games. Our results show that these algorithms represent significant advances in the state of the art of equilibrium computation for both graphical games and MAIDs.

\subsection{Related Work on Graphical Games}

In the last few years, several papers have addressed the issue of finding equilibria in structured games. For graphical games, the exact algorithms proposed so far apply only to games where the interaction structure is an undirected tree, and where each agent has only two possible actions. Kearns et al. (2001) provide an exponential-time algorithm to compute all exact equilibria in such a game, and Littman et al. (2002) provide a polynomial-time algorithm to compute a single exact equilibrium. For this very limited set of games, these algorithms may be preferable to our own, since they come with running-time guarantees. However, it is yet to be tested whether these algorithms are, in fact, more efficient in practice. Moreover, our methods are applicable to fully general games, and our results indicate that they perform well.

More effort has been focused on the computation of $\epsilon$-equilibria in general graphical games. A number of algorithms have recently been proposed for this task. Most of these use a discretized space of mixed strategies: probabilities must be selected from a grid in the simplex, which can be made arbitrarily fine. For computational reasons, however, this grid must typically be quite coarse, as the number of grid points to consider grows exponentially with the number of actions per agent. Most of these methods (implicitly or explicitly) define an equilibrium as a set of constraints over the discretized strategy space, and then use some constraint solving method: Kearns et al. (2001) use a tree-propagation algorithm (KLS); Vickrey and Koller (2002) use standard CSP variable elimination methods (VK1); and Ortiz and Kearns (2003) use arc-consistency constraint propagation followed by search (OK). Vickrey and Koller (2002) also propose a gradient ascent algorithm (VK2), and provide a hybrid refinement method that can, with further computation, reduce the equilibrium error.

As with the exact methods, the KLS algorithm is restricted to tree-structured games, and comes without experimental running time results (although it is guaranteed to run in polynomial time). Kearns et al. (2001) give a suggestion for working on a non-tree graph by constructing the junction tree and passing messages therein. However, the necessary computations are not clear and potentially very expensive. 
The VK1 algorithm is applicable to graphical games of arbitrary topology, with any number of actions per agent. It takes time exponential in the treewidth of the graph. If the treewidth is constant, then it scales linearly with the number of agents; however, our results show that it very quickly becomes infeasible if the treewidth expands (as in the grid game).

Both of these methods come with complexity guarantees, which depend on the treewidth of the graph. The others (OK and VK2, as well as our algorithm) are insensitive to treewidth - a single iteration takes time polynomial in the size of the game representation (and hence exponential only in the maximum degree of the graph). However, they all require an unknown number of iterations to converge. Corollary 7 shows that, in general, computation of equilibria with discretized strategies in games with fixed degree is hard. Thus, the lack of complexity guarantees for these methods is not surprising.

Nonetheless, experimental results for OK seem promising — they indicate that, on average, relatively few iterations are required for convergence. Results indicate that $\mathrm{OK}$ is capable of solving grid games of at least 100 agents (although in these cases $\epsilon$ was as large as 0.2 , not much better than in a random fully mixed strategy profile). However, no running time results are provided.

VK2 also exhibits strong experimental results. Vickrey and Koller (2002) have successfully found $\epsilon$-equilibria in games of up to 400 agents, with errors of up to $2 \%$ of the maximal payoff.

The main drawback to these algorithms is that they only compute $\epsilon$-equilibria. An $\epsilon$ equilibrium may be sufficient for certain applications: if the utility functions are themselves approximate, an agent certainly might be satisfied with an $\epsilon$-best response; and if we make the assumption that it is slightly costly for agents to change their minds, each agent might need an incentive greater than $\epsilon$ to deviate. However, $\epsilon$-equilibria do bring their own set of problems. The primary one is that there is no guarantee of an exact equilibrium in the neighborhood of an $\epsilon$-equilibrium. This can make it very difficult to find $\epsilon$-equilibria with small values of $\epsilon$; attempts to refine a given $\epsilon$-equilibrium may fail. The lack of a nearby Nash equilibrium also implies a certain instability. If some agent is unsatisfied with the $\epsilon$-equilibrium, play may deviate quite far from it. Finally, $\epsilon$-equilibria are more numerous than Nash equilibria (uncountably so, in general). This exacerbates the difficulty an agent faces in choosing which equilibrium to play.

The algorithms for computing $\epsilon$-equilibria are frequently faster than our own, especially when the approximations are crude or the games have more than 50 or so agents. However, the exact equilibria found by our algorithms are more satisfying solutions, and our results show that the performance of our algorithm is comparable to that of approximate methods in most cases. Surprisingly, for many games, running time results show that ours is the fastest available, particularly in the case of games with large treewidth, such as the grid game in our test cases. Furthermore, since we can use any approximate equilibrium as a starting point for our algorithm, advances in approximate methods complement our own method. The hybrid algorithm of Vickrey and Koller (2002) turns out to be unsuited to this purpose, as it tends not to remove any pure strategies from the support, but it is interesting to see whether other methods (including those listed above) might be more effective. It remains to be seen how small $\epsilon$ must be for our methods to reliably refine an approximate equilibrium. 


\subsection{Related Work on MAIDs}

Koller and Milch (2001) (KM) define a notion of dependence between agents' decisions (s-relevance), and provide an algorithm that can decompose and solve MAIDs based on this fairly coarse independence structure. Our algorithm is able to exploit finer-grained structure, resolving an open problem left by KM. In general, our method will not automatically exploit the same structure obtained by decomposing the game into its relevance components, and so our methods are best regarded as a complement to those of KM; after decomposition according to s-relevance, our algorithm can be applied to find equilibria efficiently in the decomposed problems. Running time results indicate that our methods are significantly faster than previous standard algorithms for extensive-form games. This is unsurprising, since the game representation of our test cases is exponentially larger in the number of players when converted to extensive-form.

Vickrey (2002) proposes an approximate hill-climbing algorithm for MAIDs that takes advantage of the same sort of fine-grained structure that we do: Bayesian network inference is employed to calculate expected utility as one component of the score function for a single iteration. A constraint-satisfaction approach is also proposed. However, these proposals were never implemented, so it is hard to determine what quality equilibria they would find or how quickly they would find them.

La Mura (2000) proposes a continuation method for finding one or all equilibria in a G net, a representation that is very similar to MAIDs. This proposal only exploits a very limited set of structural properties (a strict subset of those exploited by KM). This proposal was also never implemented, and several issues regarding non-converging paths seem unresolved.

Our algorithm is therefore the first to be able to exploit the finer-grained structure of a MAID. Moreover, our algorithm, applied in conjunction with the decomposition method of $\mathrm{KM}$, is able to take advantage of the full known independence structure in a MAID. A potential drawback is the requirement that strategies be $\epsilon$-perturbed. However, decreasing $\epsilon$ incurs no additional computational cost, although there are limits imposed by machine precision. Perfect equilibria - a highly desirable refinement of Nash equilibria, defined to be the limit of a sequence of $\epsilon$-perturbed equilibria as $\epsilon$ goes to zero - can therefore be computed effectively by our algorithm with little or no additional computational cost. In this sense, our use of perturbed strategies is advantageous. We have not implemented a local search algorithm to find an exact perfect equilibrium in the neighborhood of a found $\epsilon$-perturbed equilibrium, although it should be straightforward to do so.

\subsection{Conclusion and Further Work}

We have presented two related algorithms for computing exact equilibria in structured games. Our algorithms are based on the methods of GW, but perform the key computational steps in their methods much more efficiently by exploiting game structure. Our approach yields the first exact algorithm to take advantage of structure in general graphical games and the first algorithm to take full advantage of the independence structure of a MAID. These algorithms are capable of computing exact equilibria in games with large numbers of agents, which were previously intractable for exact methods. 
Our algorithms come without theoretical running time bounds, but we have noticed certain interesting trends. In both the graphical game and the MAID version of our algorithm, each iteration executes in time polynomial in the number of agents, so we have examined the number of iterations required for convergence. Our adaptive step size technique decreases the number of random restarts required to find an equilibrium, but increases the number of iterations required to cross a support cell in larger games. When adaptive step size is disabled, we have noticed that the number of iterations required, averaged across games with random payoffs, seems to grow approximately linearly. Intuitively, it makes sense that the number of iterations should be at least linear: starting from a pure strategy profile, a linear number of actions (in the number of agents) must enter the support in order for us to reach a general strategy profile. Each support boundary requires at least one iteration of our algorithm. It is somewhat surprising, however, that the number of iterations required does not grow more quickly. It is an interesting open problem to analyze the number of iterations required for convergence.

In very large games, the tendency of our algorithm to cycle increases. This phenomenon can be attributed, partially, to the cumulative effect of "wobbling": after a great number of wobbles, it is possible that the path has been altered sufficiently that it does not pass through an equilibrium. We have noticed that some games seem intrinsically harder than others, requiring many random restarts before convergence. For very large games, the overall running time of our algorithm is therefore quite unpredictable.

Our algorithms might be improved in a number of ways. Most importantly, the continuation method would profit greatly from more sophisticated path-following methods; in a number of cases, cont or MAID cont failed to find an equilibrium because it strayed too far from the path. Better path-following techniques might greatly increase the reliability of our algorithms, particularly if they obviated the need for "wobbles," which negate GW's theoretical guarantee of the convergence of the continuation method.

There are also a number of theoretical questions about the algorithms of GW that remain unresolved. Nothing is known about the worst-case or average-case running time of IPA, and no theoretical bounds exist on the number of iterations required by cont. It is interesting to speculate on how the choice of perturbation ray might affect the execution of the algorithm. Can the algorithm be directed toward particular equilibria of interest either by a careful selection of the perturbation ray or by some change in the continuation method? Is there a way of selecting perturbation rays such that all equilibria will be found? Is there a way of selecting the perturbation ray so as to speed up the execution time?

Several improvements might be made to MAID cont. We have not adapted IPA for use in MAIDs, but it should be possible to do so, making use of the generalized Lemke algorithm of Koller, Megiddo, and von Stengel (1996) to solve intermediate linearized MAIDs. The computation of $\nabla V^{G}$ might also be accelerated using a variant of the all-pairs clique tree algorithm that only computes the potentials for pairs of sepsets - sets of variables shared by adjacent cliques - rather than pairs of cliques.

Our work suggests several interesting avenues for further research. In fact, after the initial publication of these results (Blum, Shelton, \& Koller, 2003), at least one further application of our techniques has already been developed: Bhat and Leyton-Brown (2004) have shown that an adaptation of cont can be used to efficiently solve a new class of structured games called action-graph games (a generalization of local effect games as presented 
in Leyton-Brown \& Tennenholtz, 2003). We believe that these games, and other structured representations, show great promise as enablers of new applications for game theory. They have several advantages over their unstructured counterparts: they are well-suited to games with a large number of agents, they are determined by fewer parameters, making it feasible for human researchers to fully specify them in a meaningful way, and their built-in structure makes them a more intuitive medium in which to frame structured, real-world scenarios. However, to avoid the computational intractability of the general problem, each new class of structured games requires a new algorithm for equilibrium computation. We hypothesize that cont and IPA are an excellent starting point for addressing this need.

Acknowledgments. This work was supported by ONR MURI Grant N00014-00-1-0637, and by Air Force contract F30602-00-2-0598 under DARPA's TASK program. Special thanks to Robert Wilson, for kindly taking the time to guide us through the details of his work with Srihari Govindan, and to David Vickrey, for aiding us in testing our algorithms alongside his. We also thank the anonymous referees for their helpful comments. 


\section{Appendix A. Table of Notation}

\begin{tabular}{|c|c|}
\hline \multicolumn{2}{|c|}{ Notation for all games } \\
\hline$N$ & set of agents \\
\hline$\sigma_{n}$ & strategy for agent $n$ \\
\hline$\Sigma_{n}$ & strategy space for agent $n$ \\
\hline$\sigma$ & strategy profile \\
\hline$\Sigma$ & space of strategy profiles \\
\hline$\sigma_{-n}$ & strategy profile $\sigma$ restricted to agents other than $n$ \\
\hline$\Sigma_{-n}$ & space of strategy profiles for all agents other than $n$ \\
\hline$\left(\sigma_{n}, \sigma_{-n}\right)$ & $\begin{array}{l}\text { strategy profile in which agent } n \text { plays strategy } \sigma_{n} \text { and all other agents act } \\
\text { according to } \sigma_{-n}\end{array}$ \\
\hline$G_{n}(\sigma)$ & expected payoff to agent $n$ under strategy profile $\sigma$ \\
\hline$V^{G}(\sigma)$ & vector deviation function \\
\hline$R$ & retraction operator mapping points to closest valid strategy profile \\
\hline$F$ & continuation method objective function \\
\hline$\lambda$ & scale factor for perturbation in continuation method \\
\hline $\boldsymbol{w}$ & free variable in continuation method \\
\hline \multicolumn{2}{|c|}{ Notation for normal-form games } \\
\hline$a_{n}$ & action for agent $n$ \\
\hline$A_{n}$ & set of available actions for agent $n$ \\
\hline$a$ & action profile \\
\hline$A$ & set of action profiles \\
\hline$a_{-n}$ & action profile $\boldsymbol{a}$ restricted to agents other than $n$ \\
\hline$A_{-n}$ & space of action profiles for agents other than $n$ \\
\hline \multicolumn{2}{|c|}{ Notation for extensive-form games } \\
\hline$z$ & leaf node in game tree (outcome) \\
\hline$Z$ & set of outcomes \\
\hline$i$ & information set \\
\hline$I_{n}$ & set of information sets for agent $n$ \\
\hline$A(i)$ & set of actions available at information set $i$ \\
\hline$H_{n}(y)$ & sequence (history) for agent $n$ determined by node $y$ \\
\hline$Z_{h}$ & set of outcomes consistent with sequence (history) $h$ \\
\hline$b(a \mid i)$ & probability under behavior profile $b$ that agent $n$ will choose action $a$ at $i$ \\
\hline$\sigma_{n}(z)$ & realization probability of outcome $z$ for agent $n$ \\
\hline \multicolumn{2}{|c|}{ Notation for graphical games } \\
\hline $\mathrm{Fam}_{n}$ & set of agent $n$ and agent $n$ 's parents \\
\hline$\Sigma_{-n}^{f}$ & strategy profiles of agents in $\mathrm{Fam}_{n}$ other than $n$ \\
\hline$A_{-n}^{f}$ & space of action profiles of agents in $\mathrm{Fam}_{n}$ other than $n$ \\
\hline \multicolumn{2}{|c|}{ Notation for MAIDs } \\
\hline$D_{n}^{i}$ & decision node with index $i$ belonging to agent $n$ \\
\hline$U_{n}^{i}$ & utility node with index $i$ belonging to agent $n$ \\
\hline$P a_{X}$ & parents of node $X$ \\
\hline $\operatorname{dom}(S)$ & joint domain of variables in set $S$ \\
\hline
\end{tabular}




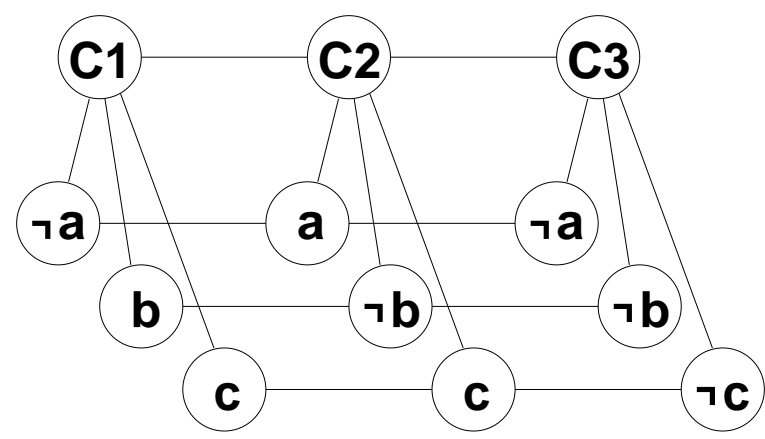

Figure 12: Reduction of the 3SAT instance $(\neg a \vee b \vee c) \wedge(a \vee \neg b \vee c) \wedge(\neg a \vee \neg b \vee \neg c)$ to a graphical game.

\section{Appendix B. Proof of Theorem 6}

Proof. The proof is by reduction from 3SAT. For a given 3SAT instance, we construct a graphical game whose equilibria encode satisfying assignments to all the variables.

Let $\boldsymbol{C}=\left\{c_{1}, c_{2}, \ldots, c_{m}\right\}$ be the clauses of the 3SAT instance in question, and let $\mathcal{V}=$ $\left\{v_{1}, \neg v_{1}, v_{2}, \neg v_{2}, \ldots, v_{n}, \neg v_{n}\right\}$ be the set of literals. If a variable appears in only one clause, it can immediately be assigned so as to satisfy that clause; therefore, we assume that variables appear in at least two clauses.

We now construct the (undirected) graphical game. For each clause, $c_{i}$, we create an agent $C_{i}$ connected to $C_{i-1}$ and $C_{i+1}$ (except $C_{1}$ and $C_{m}$, which only have one clause neighbor). We also create agents $V_{i}^{\ell}$ for each literal $\ell$ in $c_{i}$ (there are at most 3). If, for example, $c_{i}$ is the clause $\left(\neg v_{1} \vee v_{2}\right)$, it has agents $V_{i}^{\neg v_{1}}$ and $V_{i}^{v_{2}}$. We connect each of these to $C_{i}$. For every variable $v$, we group all agents $V_{i}^{v}$ and $V_{j}^{\neg v}$ and connect them in a line, the same way we connected clauses to each other. The order is unimportant.

Clause agents now have at most 5 neighbors (two clauses on either side of them and three literals) and literal agents have at most 3 neighbors (two literals on either side of them and one clause). This completely specifies the game topology. As an example, Figure 12 shows the graphical game corresponding to the 3SAT problem $(\neg a \vee b \vee c) \wedge(a \vee \neg b \vee c) \wedge(\neg a \vee \neg b \vee \neg c)$.

Now we define the actions and payoff structure. Each agent can be interpreted as a Boolean variable, and has two actions, true and false, which correspond to the Boolean values true and false. Intuitively, if a clause $C_{i}$ plays true, it is satisfied. If an agent $V_{i}^{v}$ plays true, where $v$ is a non-negated variable, then $v$ is assigned to be true. If $V_{j}^{\neg v}$ plays true, then $v$ is assigned to be false.

The payoff matrix for a clause agent $C_{i}$ is designed to ensure that if one clause is unsatisfied, the entire 3SAT instance is marked as unsatisfied. It can best be expressed in pseudo-code, as follows:

if any of $C_{i}$ 's clause neighbors play false then

payoff is $\begin{cases}1 & \text { for playing false } \\ 0 & \text { for playing true }\end{cases}$

else if at least one of $C_{i}$ 's literals plays true $\left(C_{i}\right.$ is satisfied) then 


$$
\begin{aligned}
& \text { payoff is } \begin{cases}2 & \text { for playing false } \\
2 & \text { for playing true }\end{cases} \\
& \text { else } \\
& \quad\left(C_{i} \text { is unsatisfied }\right) \\
& \text { payoff is } \begin{cases}1 & \text { for playing false } \\
0 & \text { for playing true }\end{cases} \\
& \text { end if }
\end{aligned}
$$

The payoff matrix for a literal agent $V_{i}^{\ell}$ is designed to encourage agreement with the other literals along the line for the variable $v(\ell)$ associated with $\ell$. It can be described in pseudo-code as follows:

if the parent clause $C_{i}$ plays false then

payoff is $\begin{cases}1 & \text { for playing consistently with a false assignment to } v(\ell) \\ 0 & \text { for playing the opposite }\end{cases}$

else if $V_{i}^{\ell}$ 's literal neighbors all play consistently with a single assignment to $v(\ell)$ then

payoff is $\begin{cases}2 & \text { for playing consistently with neighbors } \\ 0 & \text { for playing the opposite }\end{cases}$

else

payoff is $\begin{cases}2 & \text { for playing consistently with a false assignment to } v(\ell) \\ 0 & \text { for playing the opposite }\end{cases}$

\section{end if}

If the formula does have a satisfying assignment, then there is a pure equilibrium in which each literal is consistent with the assignment and all clauses play true; in fact, all agents receive higher payoffs in this case than in any other equilibrium, so that satisfying assignments correspond to equilibria with maximum social welfare.

If the parent clauses all play false, then clearly at equilibrium all non-negated literals must play false and all negated literals must play true. This is the trivial equilibrium. It remains to show that the trivial equilibrium is the only equilibrium for unsatisfiable formulas, i.e. that any non-trivial equilibrium can be used to construct a satisfying assignment. We first prove two simple claims.

Claim 11.1. In any Nash equilibrium, either all clauses play true with probability one or all clauses play false with probability one.

Proof. In no case is it advantageous for a clause to choose true over false, and if a neighbor clause takes the action false, it is in fact disadvantageous to do so. Thus, if any clause has a non-zero probability of playing false at an equilibrium, its neighbors, and consequently all other clauses, must play false with probability one. Therefore, the only possible equilibria have all clauses playing false or all clauses playing true.

It follows immediately from this claim that every non-trivial equilibrium has all clauses playing true with probability one.

Claim 11.2. In any non-trivial Nash equilibrium, in a line of literals for the same variable $v$, all those literals that play pure strategies must choose them consistently with a single assignment to $v$. 
Proof. Since the equilibrium is non-trivial, all clauses play true. Suppose that one of the literals, $V^{\ell}$, employs the pure strategy corresponding to a false assignment to $v$. It suffices to show that in fact all literals in the line must have pure strategies corresponding to a false assignment to $v$. Consider a neighbor $V^{\ell^{\prime}}$ of $V^{\ell}$. Either $V^{\ell^{\prime}}$ 's neighbors (one of which is $V^{\ell}$ ) both play consistently with a false assignment to $v$, in which case $V^{\ell}$ must also play consistently with a false assignment to $v$, or its neighbors play inconsistently, in which case the else clause of $V^{\ell}$ 's payoff matrix applies and $V^{\ell^{\prime}}$ must, again, play consistently with a false assignment to $v$. We may proceed all the way through the line in this manner. All literals in the line must therefore have pure strategies consistent with a false assignment to $v$, so there can be no contradicting literals.

Suppose we have a non-trivial equilibrium. Then by Claim 11.1, all clauses must play true with probability 1 . If all of the literals have pure strategies, it is clear that the equilibrium corresponds to a satisfying assignment: the literals must all be consistent with an assignment by Claim 11.2, and the clauses must all be satisfied. Some subtleties arise when we consider mixed strategy equilibria.

Note first that in each clause, the payoff for choosing true is the same as for choosing false in the case of a satisfying assignment to its literals, and is less in the case of an unsatisfying assignment. Therefore, if there is any unsatisfying assignment with non-zero probability, the clause must play false.

Consider a single clause $C_{i}$, assumed to be choosing true at equilibrium. The mixed strategies of $C_{i}$ 's literals induce a distribution over their joint actions. Because $C_{i}$ plays true, each joint action with non-zero probability must satisfy $\bigvee_{\ell} V_{i}^{\ell}$. If a literal $V_{i}^{\ell}$ has a mixed strategy, consider what will happen if we change its strategy to either one of the possible pure strategies (true or false). Some of the joint actions with non-zero probability will be removed, but the ones that remain will be a subset of the originals, so will still satisfy $\bigvee_{\ell} V_{i}^{\ell}$. Essentially, the value of $\ell$ does not affect the satisfiability of $C_{i}$, so it can be assigned arbitrarily.

Thus, if each literal in a line for a certain variable has a mixed strategy, we can assign the variable to be either true or false (and give each literal in the line the corresponding pure strategy) without making any of the clauses connected to these literals unsatisfied. In fact, we can do this if all literals in a line that have pure strategies are consistent with each other: if there are indeed literals with pure strategies, we assign the variable according to them. And by Claim 11.2, this will always be the case.

We observe briefly that this constructed graphical game has only a finite number of equilibria, even if peculiarities in the 3SAT instance give rise to equilibria with mixed strategies. If all clauses play false, then there is only one equilibrium. If all clauses play true, then we can remove them from the graph and trim the payoff matrices of the literals accordingly. Each line of literals is in this case a generic graphical game, with a finite set of equilibria. The equilibria of the original game must be a subset of the direct product of these finite sets. 


\section{References}

Bhat, N. A. R., \& Leyton-Brown, K. (2004). Computing Nash equilibria of action-graph games. In Proceedings of the Twentieth International Conference on Uncertainty in Artificial Intelligence.

Blum, B., Shelton, C., \& Koller, D. (2003). A continuation method for Nash equilibria in structured games. In Proceedings of the Eighteenth International Joint Conference on Artificial Intelligence, pp. 757-764.

Chu, F., \& Halpern, J. (2001). On the np-completeness of finding an optimal strategy in games with common payoff. International Journal of Game Theory, 30, 99-106.

Codenotti, B., \& Stefankovic, D. (2005). On the computational complexity of nash equilibria for $(0,1)$ bimatrix games. Information Processing Letters, 94, 145-150.

Conitzer, V., \& Sandholm, T. (2003). Complexity results about Nash equilibria. In Proceedings of the Eighteenth International Joint Conference on Artificial Intelligence, pp. $765-771$.

Cowell, R. G., Dawid, A. P., Lauritzen, S. L., \& Spiegelhalter, D. J. (1999). Probabilistic Networks and Expert Systems. Springer-Verlag.

Fudenberg, D., \& Tirole, J. (1991). Game Theory. The MIT Press.

Gilboa, I., \& Zemel, E. (1989). Nash and correlated equilibria: Some complexity considerations. Games and Economic Behavior, 1, 80-93.

Govindan, S., \& Wilson, R. (2002). Structure theorems for game trees. Proceedings of the National Academy of Sciences, 99(13), 9077-9080.

Govindan, S., \& Wilson, R. (2003). A global Newton method to compute Nash equilibria. Journal of Economic Theory, 110, 65-86.

Govindan, S., \& Wilson, R. (2004). Computing Nash equilibria by iterated polymatrix approximation. Journal of Economic Dynamics and Control, 28, 1229-1241.

Gül, F., Pearce, D., \& Stachetti, E. (1993). A bound on the proportion of pure strategy equilibria in generic games. Mathematics of Operations Research, 18, 548-552.

Howard, R. A., \& Matheson, J. E. (1984). Influence diagrams. In Howard, R. A., \& Matheson, J. E. (Eds.), Readings on the Principles and Applications of Decision Analysis, Vol. 2, pp. 719-762. Strategic Decision Group. article dated 1981.

Kearns, M., Littman, M. L., \& Singh, S. (2001). Graphical models for game theory. In Proceedings of the Seventeenth International Conference on Uncertainty in Artificial Intelligence, pp. 253-260.

Kohlberg, E., \& Mertens, J.-F. (1986). On the strategic stability of equilibria. Econometrica, $54(5), 1003-1038$.

Koller, D., \& Megiddo, N. (1992). The complexity of two-person zero-sum games in extensive form. Games and Economic Bahavior, 4, 528-552.

Koller, D., Megiddo, N., \& von Stengel, B. (1996). Efficient computation of equilibria for extensive two-person games. Games and Economic Behavior, 14, 247-259. 
Koller, D., \& Milch, B. (2001). Multi-agent influence diagrams for representing and solving games. In Proceedings of the Seventeenth International Joint Conference on Artificial Intelligence, pp. 1027-1034.

Kuhn, H. W. (1953). Extensive games and the problem of information. In Contributions to the Theory of Games II, eds. H. W. Kuhn and A. W. Tucker, Vol. 28, pp. 193-216. Princeton University Press, Princeton, NJ.

La Mura, P. (2000). Game networks. In Proceedings of the Sixteenth International Conference on Uncertainty in Artificial Intelligence, pp. 335-342.

Lauritzen, S. L., \& Spiegelhalter, D. J. (1998). Local computations with probabilities on graphical structures and their application to expert systems. Journal of the Royal Statistical Society, B 50(2), 157-224.

Lemke, C. E., \& Howson, Jr., J. T. (1964). Equilibrium points in bimatrix games. Journal of the Society of Applied Mathematics, 12(2), 413-423.

Leyton-Brown, K., \& Tennenholtz, M. (2003). Local-effect games. In Proceedings of the Eighteenth International Joint Conference on Artificial Intelligence, pp. 772-777.

Littman, M. L., Kearns, M., \& Singh, S. (2002). An efficient exact algorithm for singly connected graphical games. In Advances in Neural Information Processing Systems 14, Vol. 2, pp. 817-823.

McKelvey, R. D., \& McLennan, A. (1996). Computation of equilibria in finite games. In Handbook of Computational Economics, Vol. 1, pp. 87-142. Elsevier Science.

McKelvey, R. D., McLennan, A. M., \& Turocy, T. L. (2004). Gambit: Software tools for game theory, version 0.97.07.. http://econweb.tamu.edu/gambit.

Nash, J. (1951). Non-cooperative games. The Annals of Mathematics, 52(2), 286-295.

Nudelman, E., Wortman, J., Shoham, Y., \& Leyton-Brown, K. (2004). Run the GAMUT: A comprehensive approach to evaluating game-theoretic algorithms. In Third International Conference on Autonomous Agents and Multi-Agent Systems.

Ortiz, L. E., \& Kearns, M. (2003). Nash propagation for loopy graphical games. In Advances in Neural Information Processing Systems 15, Vol. 1, pp. 793-800.

Romanovskii, I. (1962). Reduction of a game with complete memory to a matrix game. Doklady Akademii Nauk, SSSR 144, 62-64. [English translation: Soviet Mathematics 3 , pages 678-681].

Vickrey, D. (2002). Multiagent algorithms for solving structured games. Undergraduate honors thesis, Stanford University.

Vickrey, D., \& Koller, D. (2002). Multi-agent algorithms for solving graphical games. In Proceedings of the Eighteenth National Conference on Artificial Intelligence (AAAI), pp. $345-351$.

von Stengel, B. (1996). Efficient computation of behavior strategies. Games and Economic Behavior, 14, 220-246.

Watson, L. T. (2000). Theory of globally convergent probability-one homotopies for nonlinear programming. SIAM Journal on Optimization, 11(3), 761-780. 